\title{
Comprehensive identification of survival-associated genes for cancers
}

\author{
Hongde Liu' ${ }^{1 *}$, Kun Luo ${ }^{2}$, Huamei $\mathrm{Li}^{1}$, Xiao Sun ${ }^{1}$
}

${ }^{1}$ State Key Laboratory of Bioelectronics, School of Biological Science \& Medical

Engineering, Southeast University, Nanjing 210096, China

${ }^{2}$ Department of Neurosurgery, Xinjiang Evidence-Based Medicine Research Institute, First Affiliated Hospital of Xinjiang Medical University, Urumqi 830054, China

\section{*The corresponding author:}

Hongde Liu, e-mail: liuhongde@seu.edu.cn (HDL).

\section{Authors' e-mails:}

Hongde Liu: liuhongde@seu.edu.cn (HDL);

Kun Luo: luokun_2822@sohu.com (KL);

Huamei Li: li_hua_mei@163.com(HML);

Xiao Sun: xsun@seu.edu.cn(XS).

\section{Highlights:}

- The number of possible prognostic and diagnostic genes for cancers;

- A list of independent prognostic genes for each cancer;

- The universal prognostic genes mainly function in the spindle assembly checkpoint;

- Statistical links between mutated pathways and prognostic genes. 


\begin{abstract}
Prognostic signature is important in estimating cancer risk, subtyping cancer, and planning treatment. A single gene as a prognostic marker would facilitate the development of a clinical test. Here we showed that the number of prognostic and diagnostic genes differ greatly across cancers. By considering both the survival difference and the fold change of expression in cancer, we revealed the prognostic genes for each cancer and found twenty two genes with both diagnostic and prognostic capacity. The universal prognostic genes (CDC20, CDCA8, ASPM, ERCC6L, and GTSE1) mainly function in the spindle assembly checkpoint, and show more statistical links to mutated pathways, suggesting that expression of these genes can be altered by mutations from many pathways. Briefly, we systematically identified the prognostic genes and revealed the associations between the prognostic genes and genes mutated in cancer.
\end{abstract}

Keywords: Prognostic gene; Cancer; Mutation; Diagnosis 


\section{Introduction}

Cancers are characterized by distinct patterns of mutation and gene expression, associated with different prognosis.

Prognostic expression signatures have been extensively identified and overall survival exhibits links to diverse biological processes. Uhlen et al summarized that shorter survival is associated with the upregulation of genes related to cell growth and the downregulation of genes related to cellular differentiation. ${ }^{1}$ For breast cancer, the MammaPrint test, including 70 genes, is able to assess the benefit of chemotherapy. ${ }^{2}$ Metastasis is a key factor in short survival. In digestive cancers, besides activation of the mitotic cell cycle, altered expression in the extracellular matrix is linked to poor prognosis. ${ }^{3-5}$ A 64-gene signature is associated with metastasis for non-small cell lung carcinoma (NSCLC). ${ }^{6}$ Inflammatory-related genes are also involved in prognosis in glioblastoma (GBM), ${ }^{7}$ colorectal, and pancreatic cancers. ${ }^{3,4}$ Recently, an estimate of the risk of recurrence of colon cancer was shown to be provided by the total tumor-infiltrating T-cell count and the cytotoxic tumor-infiltrating T-cell count. ${ }^{8}$ In acute myeloid leukemia (AML), the worst overall survival is associated with the expression of multidrug resistant genes. ${ }^{9}$ Also, genes in the tumor microenvironment have a prognostic role for NSCLC. ${ }^{10}$

Cancer-associated mutations have also been extensively studied. Recently, 299 driver mutation genes were revealed. ${ }^{11}$ The study confirmed that microsatellite instability was associated with an improved response to immune checkpoint therapy. ${ }^{11}$ A model estimating the mutation load of 24 genes predicted the response to cancer immunotherapy with anti-CTLA-4 and anti-PD-1 treatments. ${ }^{12}$

The present work focuses on two questions. Firstly, what are the independent prognostic genes for each cancer? The expression signatures in the literature are actually combinations of a set of genes. ${ }^{2-7,10,13}$ A single gene as a prognostic marker would facilitate the development of a clinical test. Although some independent prognostic genes have been identified, including the genes for PSA for prostate cancer, ${ }^{14}$ microRNA-148a for bladder cancer, ${ }^{15}$ telomerase for colorectal cancer, ${ }^{16}$ KIAA1199 for NSCLC, $^{17}$ and CTHRC1 for gastric cancer, $^{18}$ a systems-level identification of an independent prognostic gene is still needed. We also noticed that the signature genes differ depending on the criteria. It will be interesting to discover which genes are identified under tighter criteria. Secondly, what are the links between expression of the prognostic genes and the mutations present for a specific cancer? Given that the mutations are the original driving factors for the tumor, ${ }^{19}$ what is the link from the 
mutations to the altered gene expression, and how does the link determine the prognosis? We will explore the connection.

Here we revealed the independent overall survival-associated genes for each cancer type and identified the genes with capacities of both prognosis and diagnosis. The links between mutation and the prognostic gene expression were also investigated.

\section{Materials and methods}

\subsection{Datasets}

Gene expression data, survival data, and mutation data were retrieved from TCGA project from the initial release of Genomic Data Commons (GDC) in October 2016 using RTCGAToolbox. ${ }^{20}$ A total of 9523 samples across 29 tumor types were downloaded, including 8811 tumor tissues and 712 non-tumor tissues. The abbreviation for cancer type is in supplementary information. Microarray-based gene expression data (gene expression omnibus ID: GSE21501 for pancreatic cancer ${ }^{21}$ ) were retrieved for validating.

\subsection{Identification of the prognostic genes}

The prognostic genes were identified with a log-rank test in a Kaplan-Meier survival model. In each cancer type, for each gene, patients were classified into two groups, the high-expression group $(\mathrm{H})$ and the low-expression group $(\mathrm{L})$, using the expression median of the gene as a cutoff. In identifying, we considered both survival difference (P[SV]) and the expression change $(\mathrm{FC}(\mathrm{H} / \mathrm{L}))$ between the two groups. The area under the curve (AUC) of a receiver operating characteristic (ROC) curve and the expression fold change between the cancer $(\mathrm{C})$ and normal $(\mathrm{N})$ tissues $(\mathrm{FC}(\mathrm{C} / \mathrm{N}))$ were employed to indicate the diagnosis ability.

\subsection{Regression for the expression of the prognostic genes with the mutation counts}

The 40 prognostic genes, which were identified with $\mathrm{P}[\mathrm{SV}] \leq 10^{-6}$ and $\mathrm{FC}[\mathrm{H} / \mathrm{L}] \geq 4$, and the top 200 frequently mutated genes, were included in this section. Firstly, for each prognostic gene, the dependence between its expression and the mutation counts of the 200 mutated genes were tested with a chi-squared $\left(\chi^{2}\right)$ test for each cancer. The mutated genes with $p \leq 0.001\left(\chi^{2}\right.$ test) were included in an enrichment analysis. A cutoff of $p \leq 0.05$ was used to find the enriched terms and pathways. Upon satisfaction of those criteria, a link between the prognostic gene and the mutated pathway (terms) was counted. This was done for all cancers to see how many cancer types shared the link. Secondly, we carried out a generalized linear regression of the expression of the prognostic gene with the mutation counts of the top 200 frequently mutated genes for each cancer type. The regression generated a set of parameters indicating the contribution of the mutation in explaining the expression level of the prognostic 
gene. Only mutated genes with a significant parameter were used to construct a network. More details are in Supplementary Information.

\section{Results}

\subsection{Prognostic genes differs across cancers}

First, we found under the same cutoff of overall survival difference (P[SV]), the number of potential prognostic genes differed between cancer types (Fig. 1A and Fig. S1A). Cancer CHOL, ESCA, STAD, COAD, GBM and PCPG had only a limited number of prognostic genes (Fig. 1A). We assessed the diagnostic genes by calculating the area under the receiver operating characteristic (ROC) curve (AUC) between the cancer and control (normal) data. The cancers also had different numbers of genes at a high AUC value (AUC $\geq 0.9$ ) (Fig. 1A). The result suggests that the number of prognostic and diagnostic genes differs between cancers.

We did not observe a link between the number of prognostic genes and the survival probability (Fig. 1B). We calculated Pearson correlation coefficients (PCCs) of the gene expression of 25,301 genes for each pair of patients for each cancer, and associated the coefficients to the five-year survival probability. Interestingly, the survival probability highly correlated to the standard deviation (Std) of the PCC (r=-0.97) (Fig. 1C).

The pathways and gene ontology (GO) terms that were enriched for the survival-related genes exhibited distinct clusters for the cancer types (Fig. 1D). In KIRP, ACC, and MESO, the genes were enriched for the terms “cell cycle” and “cell division”. CESC, BRCA, STAD, BLCA, and HNSC shared enriched term "T cell costimulation”. LUSC, THCA, DBLC, UCEC, USC, and LIHC had common terms of “cell adhesion”. GBM, LAML, and PAAD were not included in any of the clusters mentioned above. We observed a substantial enrichment in the term "Glycoprotein". P-glycoprotein was found in the multidrug resistance (MDR) phenotype of adult solid tumors. ${ }^{22}$ The enrichment for the genes whose expressions change greatly is shown in Fig. S1B.

\subsection{Prognostic genes identified}

Then, we identified the prognostic genes. We considered two factors to be important. The first was a tight association between the variation of clinical outcome (here, overall survival) and the variation of expression level of the gene, which can be represented by the survival difference (P[SV]) between the high- and low-expression groups. The second was the discriminability of the gene by mRNA level between the two groups. The fold change of gene expression in the high $(\mathrm{H})$ and low $(\mathrm{L})$ groups $(\mathrm{FC}(\mathrm{H} / \mathrm{L})$ ) was used to indicate such 
discriminability.

First, relaxed criteria of $\mathrm{P}[\mathrm{SV}] \leq 10^{-3}$ and $\mathrm{FC}(\mathrm{H} / \mathrm{L}) \geq 2$, selecting the top 10 genes for each cancer type, resulted in 236 genes in 29 cancer types (Fig. S2A, Suppl-1). Eight of the 236 genes were in the list identified in literature by $\mathrm{P}[\mathrm{SV}] \leq 10^{-3}$ in 17 cancers $^{1}$ (Fig. S2B). The genes include C1orf88 for ACC, BCL2L14 for BLCA, TMEM65 for BRCA, RBM38 for CESC, ATP13A3 for CHOL, ATOH1 for CORD, ATP1A3 for DLBC and UCS, GRPEL2 for ESCA, RARRES2 for GBM, CHGB for HNSC, CLDN3 for KICH, ATP6V1C2 for KIRH, HOXD10 for KIRP, TREML2 for LAML, ISL2 for LGG, CDC20 for LIHC, GTSE1 for LUAD, PAPPA for LUSC, CEP55 for MESO, DYDC2 for OV, MYEOV for PAAD, KIAA0319 for PRAD, LBH for STAD, CILP for THCA, PRKCB for THYM, and TP53TG3B for UCEC (Suppl-1). Very few genes were shared across cancer types, consistent with the literatures. ${ }^{1,23}$ It is notable that the subunits of P- and V-ATPases (such as ATP13A3, ATP1A3, and ATP6V1C2) were in the list. These genes are responsible for transporting cations across membranes and organelle acidification. ${ }^{24}$

Then, the 236 genes were filtered further with stricter criteria, $\mathrm{P}[\mathrm{SV}] \leq 10^{-6}$ and $\mathrm{FC}(\mathrm{H} / \mathrm{L}) \geq 4$, resulting in a list of 40 genes (Fig. 2A and Suppl-2). Cancers CHOL, ESCA, TGCT, OV and UCS had no prognostic genes under the criteria (Fig. 2A). The genes CDC20, CDCA8, and CEP55 were prognostic in more than three cancer types. Other genes were specific for particular cancers, such as MYEOV for PAAD. Most of the genes had a hazard effect, meaning that high expression of the gene was associated with poorer overall survival (Fig. 2A). The prognostic genes were enriched for the terms of cell cycle, cell division, and cytoskeleton (Fig. S2 C and D). Moreover, in the space of the principal components (PCs) constructed with expression of the 40 genes, the cancer tissues showed clustering patterns and cancer types could be discriminated (Fig. 2B), indicating that the genes represent cancer-type specific survival information.

As few prognostic genes were identified for some cancer types with the strict criteria (Fig. 2A), another strategy, using the Pearson correlation coefficient between survival time and gene expression, was applied to identify optional candidates from the 236 prognostic genes. The result is shown in Fig. 2C. Although some of these genes are not in the list in Fig. 2A, they performed well in indicating survival, for instance, HIST3H2A and LRRC61 for GBM, and CA11 for PAAD (Fig. 2C and D).

Some of the prognostic genes have been previously identified. For instance, lower expression of MYBL2 is associated with a favorable overall survival in $\mathrm{LGG}^{25} \mathrm{LIHC}^{26}$ and NSCLC. ${ }^{27}$ 
High DKK1 was identified for gastric cancer. ${ }^{28}$ NPTX2 has been suggested to have prognostic value for $G B M,{ }^{29}$ we found it was moderately significant $(\mathrm{p} \leq 0.05)$ for survival. ${ }^{30}$ We also randomly selected one gene, MYEOV, and tested its prognostic performance with microarray-based data for PAAD (Fig. S2E). ${ }^{21}$

\subsection{Genes with prognostic and diagnostic capacities}

We noticed that although some of the genes have prognostic abilities, their average expression levels are even higher in normal tissue than in the low-expression cancer tissues, which leads to confusion in predicting clinical outcome because there is a difficulty in deciding if an unknown tissue is cancer. An example is from gene DKK1 for LUAD (Fig. S3).

Therefore, we assessed the diagnostic ability of the 236 prognostic genes from two aspects. One was the fold change of gene expression between the cancer $(\mathrm{C})$ and the control $(\mathrm{N})$ tissues $(\mathrm{FC}(\mathrm{C} / \mathrm{N}))$, and the other was the AUC in diagnosis. With the criteria $\left|\log _{2} \mathrm{FC}(\mathrm{C} / \mathrm{N})\right| \geq 1.5$ and AUC $\leq 0.8$, we identified 22 genes (Fig. 3A, Fig. S4, Suppl-3). For CDC20, CDCA8, CDK1, MYBL2, KIF14, SPAG5, and STC2, their prognostic value has been previously identified. We here confirmed their diagnostic value. For others, we revealed both prognostic and diagnostic values. As shown in Fig. 3B, the expression levels of the genes exhibited a successive increase from the controls, to the low-expression cancer group, and then to the high-expression cancer group, making assessment of both diagnosis and prognosis possible. The genes CDC20, CDCA8, MYBL2, C1QTNF6, CEP55, CDK1, and KIF14 are universal, since they mark multiple types of cancers not only in prognosis but also in diagnosis. CDC20 can be a prognostic marker for LIHC and KIRC, and can be a diagnostic marker for more than nine types of cancer (Fig. S5). The protein encoded by CDC20 is required for the full ubiquitin ligase activity of the anaphase promoting complex/cyclosome (APC/C). The spindle assembly checkpoint causes CDC20 to bind to different sites on the APC/C, which alters APC/C substrate specificity. ${ }^{31}$ The product of CDCA8 is a component of the vertebrate chromosomal passenger complex (CPC), which ensures correct chromosome alignment and segregation and is required for microtubule stabilization and spindle assembly. ${ }^{32}$ CDK1 and MYBL2 are central regulators of cell cycle progression. ${ }^{33,34}$ MYBL2 is a well-known prognostic predictor. ${ }^{33}$ KIF14 is involved in many processes, including chromosome segregation and mitotic spindle formation. ${ }^{35,36}$

We also tested the possibility of fifteen immunoregulation-related genes in prognosis as well as diagnosis (Fig. 4). PVR was significant for prognosis for KIRC and HNSC. The product of $P V R$ is the ligand of TIGIT, which can repress the activity of NK cells. ${ }^{37}$ CD48 has prognostic 
value for BRCA (Fig. 4).

Briefly, we identified 22 genes that have both prognostic and diagnostic capacities.

\subsection{Links between mutations and prognostic genes}

Finally, we investigated the association between the mutations and the expression of the prognostic genes. The genes with mutations were identified (Fig. S6A and B). The total mutations did not seem to be directly associated with survival (Fig. S6C). We normalized the mutation rate by dividing by gene length, as a longer gene has more chances to mutate. ${ }^{19}$ Interestingly, we found that genes with $\sim 1$ mutation per kbp had the lowest expression, in both cancer and control samples (Fig. S6D). Additional analysis is shown in Fig. S7.

We revealed the link between the mutated pathway and the expression of the prognostic genes (Fig. 5A). The prognostic genes could be placed in three classes. In the first class, the gene expression was affected by many mutated pathways in more than five cancer types. These genes were CDC20, CDCA8, ASPM, ERCC6L, KLRA1, KIF14, SGOL1, and FAM72D. We noticed CDC20, ERCC6L, ASPM, and CDCA8 are related to anaphase spindle assembly. ${ }^{31,32,38,39}$ The second class included the genes whose expression was affected by only a few pathways, such "Focal adhesion", the "FoxO and ErbB signaling pathways" and "Carbohydrate digestion and absorption”. These genes included GTS1, C1orf88, C5orf32, ATP6V1C2, CLIP, and C1QTNF6. In the last class, links were found to less than three cancer types, showing specificity. The genes MYEOV, ANKRD56, and C7orf29 are connected to mutations in the "Tight junction” and “Long-term potentiation” pathways. Mutations occurring in the PI3K/PI4K domain, methylation-related and central carbon metabolism-related genes showed extensive alteration of the expression levels of the prognostic genes.

We then tested the relationships between the mutated genes and the prognostic genes (Fig. 5B). CDC20, $C D C A 8$, and $A S P M$ were associated with a greater number of mutated genes. Mutations in PKHD1, ATM, and ZNF536 were associated with a greater number of prognostic genes.

Frequently mutated genes, including TP53 and PTEN, showed a strong association to CDC20 expression (Fig. 5B, Fig. 2A). Mutations in TG (thyroglobulin), EP400, a component of the NuA4 histone acetyltransferase complex, and SI (a sucrase-isomaltase enzyme) showed links with CDCA8. Mutations in CNTNAP5, which encodes a cell adhesion molecule in the nervous system, ${ }^{40}$ and mutations in ATM, whose product belongs to the PI3/PI4-kinase family and functions as a cell cycle checkpoint kinase, exhibited a link to the prognostic gene ASPM. GTSE1 encodes a protein that is involved in p53-induced cell cycle arrest in G2/M phase by 
interfering with microtubule rearrangements. ${ }^{41}$ We found that mutations in cadherin 23 (CDH23), which helps cells stick together, and TEX15, which is involved in DNA double-stranded break repair, are linked to GTSE1 expression. The expression of MYEOV is mainly affected by mutations in genes associated with intraflagellar transport (DYNC2H1), actin-microtubule interactions and cellular junctions (MACF1), myotendinous junctions (COL22A1), and calcium-binding microfibrils and glucose homeostasis (FBN1).

Briefly, we have provided an insight into the relationships between prognostic genes and genes mutated in cancers.

\section{Discussion}

Prognostic genes are important in estimating low-risk patients, assessing cancer progression, subtyping cancer, and making a proper plan for medical treatment. Here, we reveal the prognostic genes for overall survival for 29 cancers by systematic scanning.

Three points are highlighted. Firstly, the prognostic genes vary greatly among the cancer types. It seems that the more subtypes the cancer has, the fewer prognostic genes. For instance, GBM can be classified into six subgroups (IDH, K27, G34, RTK I and II, and MES). ${ }^{42}$ Breast tumors have five molecular subtypes (Luminal A, Luminal B, Her2 overexpressing, basal, and normal-like). ${ }^{43}$ ESCA includes four subtypes. ${ }^{44}$ The three cancers had fewer prognostic genes (Fig. 1A-B). In the literature, high levels of intratumor genetic heterogeneity are associated with poorer survival across cancers. ${ }^{45}$ We found a significant association between the intertumor expression heterogeneity and the overall survival (Fig. 1C).

Secondly, the 236 prognostic genes were identified under the criteria of both survival difference and expression change. Expression differentiation between cancer and adjacent normal tissues has been proven to be irrelevant to survival. ${ }^{46}$ We demonstrated it is not appropriate to identify prognostic genes by comparing gene expression between cancer and control samples (DKK1, Fig. S3). ${ }^{46}$ We identified 22 genes for both prognosis and diagnosis. High-quality prognostic genes, including CDC20, ASPM, CDCA8, SGOL1, and ERCC6L, play roles in G2/M processes, such as the spindle assembly checkpoint. ${ }^{31,32,38,39}$ Therefore, the regulation of anaphase of the cell cycle is intimately associated with patient survival.

Thirdly, we associated the mutations and the prognostic genes. Mutations in the PI3K-AKT, ErbB, and FoxO signaling pathways, and in the biological processes of tight junction and methylation, can ubiquitously alter the expression of the prognostic genes. Further relationships were seen between prognostic genes that function in anaphase of the cell cycle, especially CDC20, CDCA8, ASPM and GTSE1, and the mutated genes including TP53, PTEN, 
ATM, EP400 and BAI3. PKHD1 mutations link seven prognostic genes (Fig. 5B). Fibrocystin, encoded by PKHD1 in the liver and kidney, may be involved in cell adhesion, cell repulsion, and the growth and division of cells. ${ }^{47}$

Our results provide a comprehensive prognostic and diagnostic gene list, and reveal the characteristics of prognostic genes of cancer and the statistical association to mutation.

\section{Contributions}

HDL and KL designed the study and wrote the manuscript. HDL and HML did data analysis. KL and XS provided interpretation and discussion. All authors contributed to and approved the final manuscript.

\section{Declaration of interests}

The authors declare that they have no competing interests.

\section{Acknowledgments}

We thank the supports from the National Natural Science Foundation of China (No. 31371339 and No. 81660471) and Key Research \& Development Program of Jiangsu Province (BE2016002-3).

\section{References}

1 Uhlen, M. et al. A pathology atlas of the human cancer transcriptome. Science 357, doi:10.1126/science.aan2507 (2017).

2 Cardoso, F. et al. 70-Gene Signature as an Aid to Treatment Decisions in Early-Stage Breast Cancer. The New England journal of medicine 375, 717-729, doi:10.1056/NEJMoa1602253 (2016).

3 Birnbaum, D. J. et al. A 25-gene classifier predicts overall survival in resectable pancreatic cancer. BMC medicine 15, 170, doi:10.1186/s12916-017-0936-z (2017).

4 Barrier, A. et al. Colon cancer prognosis prediction by gene expression profiling. Oncogene 24, 6155-6164, doi:10.1038/sj.onc.1208984 (2005).

5 Nault, J. C. et al. A hepatocellular carcinoma 5-gene score associated with survival of patients after liver resection. Gastroenterology 145, 176-187, doi:10.1053/j.gastro.2013.03.051 (2013).

$6 \quad$ Lu, Y. et al. A gene expression signature predicts survival of patients with stage I non-small cell lung cancer. PLoS medicine 3, e467, doi:10.1371/journal.pmed.0030467 (2006).

7 Kim, Y. W. et al. Identification of prognostic gene signatures of glioblastoma: a study based on TCGA data analysis. Neuro-oncology 15, 829-839, doi:10.1093/neuonc/not024 (2013).

8 Pages, F. et al. International validation of the consensus Immunoscore for the classification of colon cancer: a prognostic and accuracy study. Lancet 391, 2128-2139, doi:10.1016/S0140-6736(18)30789-X (2018).

9 Wilson, C. S. et al. Gene expression profiling of adult acute myeloid leukemia identifies novel biologic clusters for risk classification and outcome prediction. Blood 108, 685-696, doi:10.1182/blood-2004-12-4633 (2006). 
Navab, R. et al. Prognostic gene-expression signature of carcinoma-associated fibroblasts in non-small cell lung cancer. Proceedings of the National Academy of Sciences of the United States of America 108, 7160-7165, doi:10.1073/pnas.1014506108 (2011).

Bailey, M. H. et al. Comprehensive Characterization of Cancer Driver Genes and Mutations. Cell 174, 1034-1035, doi:10.1016/j.cell.2018.07.034 (2018).

Lyu, G. Y., Yeh, Y. H., Yeh, Y. C. \& Wang, Y. C. Mutation load estimation model as a predictor of the response to cancer immunotherapy. NPJ genomic medicine 3, 12, doi:10.1038/s41525-018-0051-x (2018).

Abdul Aziz, N. A. et al. A 19-Gene expression signature as a predictor of survival in colorectal cancer. BMC medical genomics 9, 58, doi:10.1186/s12920-016-0218-1 (2016).

Palmberg, C., Koivisto, P., Visakorpi, T. \& Tammela, T. L. PSA decline is an independent prognostic marker in hormonally treated prostate cancer. European urology 36, 191-196, doi:10.1159/000067996 (1999).

Ma, L., Xu, Z., Xu, C. \& Jiang, X. MicroRNA-148a represents an independent prognostic marker in bladder cancer. Tumour biology : the journal of the International Society for Oncodevelopmental Biology and Medicine 37, 7915-7920, doi:10.1007/s13277-015-4688-0 (2016).

Bertorelle, R. et al. Telomerase is an independent prognostic marker of overall survival in patients with colorectal cancer. British journal of cancer 108, 278-284, doi:10.1038/bjc.2012.602 (2013).

Deng, F. et al. Overexpression of KIAA1199: An independent prognostic marker in nonsmall cell lung cancer. Journal of cancer research and therapeutics 13, 664-668, doi:10.4103/jcrt.JCRT_61_17 (2017). $\mathrm{Gu}$, L. et al. Cthrc1 overexpression is an independent prognostic marker in gastric cancer. Human pathology 45, 1031-1038, doi:10.1016/j.humpath.2013.12.020 (2014).

Vogelstein, B. et al. Cancer genome landscapes. Science 339, 1546-1558, doi:10.1126/science.1235122 (2013).

Samur, M. K. RTCGAToolbox: a new tool for exporting TCGA Firehose data. PloS one 9, e106397, doi:10.1371/journal.pone.0106397 (2014).

21 Stratford, J. K. et al. A six-gene signature predicts survival of patients with localized pancreatic ductal adenocarcinoma. PLoS medicine 7, e1000307, doi:10.1371/journal.pmed.1000307 (2010).

Leighton, J. C., Jr. \& Goldstein, L. J. P-glycoprotein in adult solid tumors. Expression and prognostic significance. Hematology/oncology clinics of North America 9, 251-273 (1995).

Wang, Y., Goodison, S., Li, X. \& Hu, H. Prognostic cancer gene signatures share common regulatory motifs. Scientific reports 7, 4750, doi:10.1038/s41598-017-05035-3 (2017).

Beyenbach, K. W. \& Wieczorek, H. The V-type H+ ATPase: molecular structure and function, physiological roles and regulation. The Journal of experimental biology 209, 577-589, doi:10.1242/jeb.02014 (2006).

Zhang, X., Lv, Q. L., Huang, Y. T., Zhang, L. H. \& Zhou, H. H. Akt/FoxM1 signaling pathway-mediated upregulation of MYBL2 promotes progression of human glioma. Journal of experimental \& clinical cancer research : CR 36, 105, doi:10.1186/s13046-017-0573-6 (2017).

Guan, Z., Cheng, W., Huang, D. \& Wei, A. High MYBL2 expression and transcription regulatory activity is associated with poor overall survival in patients with hepatocellular carcinoma. Current research in translational medicine 66, 27-32, doi:10.1016/j.retram.2017.11.002 (2018).

Fan, X. et al. B-Myb Mediates Proliferation and Migration of Non-Small-Cell Lung Cancer via Suppressing IGFBP3. International journal of molecular sciences 19, doi:10.3390/ijms19051479 (2018). 
Hong, S. A. et al. Prognostic value of Dickkopf-1 and ss-catenin expression in advanced gastric cancer. BMC cancer 18, 506, doi:10.1186/s12885-018-4420-8 (2018). Skiriute, D. et al. Promoter methylation of AREG, HOXA11, hMLH1, NDRG2, NPTX2 and Tes genes in glioblastoma. Journal of neuro-oncology 113, 441-449, doi:10.1007/s11060-013-1133-3 (2013).

Carlson, M. R. et al. Relationship between survival and edema in malignant gliomas: role of vascular endothelial growth factor and neuronal pentraxin 2. Clinical cancer research : an official journal of the American Association for Cancer Research 13, 2592-2598, doi:10.1158/1078-0432.CCR-06-2772 (2007).

Izawa, D. \& Pines, J. How APC/C-Cdc20 changes its substrate specificity in mitosis. Nature cell biology 13, 223-233, doi:10.1038/ncb2165 (2011).

Gassmann, R. et al. Borealin: a novel chromosomal passenger required for stability of the bipolar mitotic spindle. The Journal of cell biology 166, 179-191, doi:10.1083/jcb.200404001 (2004).

Musa, J., Aynaud, M. M., Mirabeau, O., Delattre, O. \& Grunewald, T. G. MYBL2 (B-Myb): a central regulator of cell proliferation, cell survival and differentiation involved in tumorigenesis. Cell death \& disease 8, e2895, doi:10.1038/cddis.2017.244 (2017).

Diril, M. K. et al. Cyclin-dependent kinase $1(\mathrm{Cdk} 1)$ is essential for cell division and suppression of DNA re-replication but not for liver regeneration. Proceedings of the National Academy of Sciences of the United States of America 109, 3826-3831, doi:10.1073/pnas.1115201109 (2012).

Zhu, C. et al. Functional analysis of human microtubule-based motor proteins, the kinesins and dyneins, in mitosis/cytokinesis using RNA interference. Molecular biology of the cell 16, 3187-3199, doi:10.1091/mbc.e05-02-0167 (2005).

Corson, T. W. et al. KIF14 messenger RNA expression is independently prognostic for outcome in lung cancer. Clinical cancer research : an official journal of the American Association for Cancer Research 13, 3229-3234, doi:10.1158/1078-0432.CCR-07-0393 (2007).

Stanietsky, N. et al. The interaction of TIGIT with PVR and PVRL2 inhibits human NK cell cytotoxicity. Proceedings of the National Academy of Sciences of the United States of America 106, 17858-17863, doi:10.1073/pnas.0903474106 (2009).

Baumann, C., Korner, R., Hofmann, K. \& Nigg, E. A. PICH, a centromere-associated SNF2 family ATPase, is regulated by Plk1 and required for the spindle checkpoint. Cell 128, 101-114, doi:10.1016/j.cell.2006.11.041 (2007).

Jiang, K. et al. Microtubule minus-end regulation at spindle poles by an ASPM-katanin complex. Nature cell biology 19, 480-492, doi:10.1038/ncb3511 (2017).

Spiegel, I., Salomon, D., Erne, B., Schaeren-Wiemers, N. \& Peles, E. Caspr3 and caspr4, two novel members of the caspr family are expressed in the nervous system and interact with PDZ domains. Molecular and cellular neurosciences 20, 283-297 (2002).

$\mathrm{Xu}$, T. et al. High G2 and S-phase expressed 1 expression promotes acral melanoma progression and correlates with poor clinical prognosis. Cancer science 109, 1787-1798, doi:10.1111/cas.13607 (2018). Sturm, D. et al. Hotspot mutations in H3F3A and IDH1 define distinct epigenetic and biological subgroups of glioblastoma. Cancer cell 22, 425-437, doi:10.1016/j.ccr.2012.08.024 (2012).

Dai, X. et al. Breast cancer intrinsic subtype classification, clinical use and future trends. American journal of cancer research 5, 2929-2943 (2015).

Cancer Genome Atlas Research, N. et al. Integrated genomic characterization of oesophageal carcinoma. Nature 541, 169-175, doi:10.1038/nature20805 (2017). 

available under aCC-BY-NC-ND 4.0 International license.

survival. Oncotarget 7, 10051-10063, doi:10.18632/oncotarget.7067 (2016).

An, N., Yu, Z. \& Yang, X. Expression Differentiation Is Not Helpful in Identifying Prognostic Genes Based on TCGA Datasets. Molecular therapy. Nucleic acids 11, 292-299, doi:10.1016/j.omtn.2018.02.013 (2018).

47 Onuchic, L. F. et al. PKHD1, the polycystic kidney and hepatic disease 1 gene, encodes a novel large protein containing multiple immunoglobulin-like plexin-transcription-factor domains and parallel beta-helix 1 repeats. American journal of human genetics 70, 1305-1317, doi:10.1086/340448 (2002). 


\section{Figure legends}

Figure 1 Survival-associated genes differ among cancers.

A. The number of genes associated with a differential survival (P[SV]), with an expression change in the cancer tissues (FC(H/L)), with a capacity of diagnosis for cancer (AUC), and with an expression change between the control $(\mathrm{N})$ and cancer $(\mathrm{C})$ samples $(\mathrm{FC}(\mathrm{C} / \mathrm{N})$ ), respectively. In each cancer type, for each gene, cancer tissues are divided into high $(\mathrm{H})$ and low (L) expression groups based on the median expression of the gene in the cancer type. $\mathrm{P}[\mathrm{SV}]$ and $\mathrm{FC}(\mathrm{H} / \mathrm{L})$ represent survival difference and fold change between the two groups, respectively. $\mathrm{FC}(\mathrm{C} / \mathrm{N})$ means the fold change between the cancer tissues and normal controls. AUC is the area under receiver operating characteristic (ROC) curves in diagnosing the cancer samples with expression of the gene.

B. The number of prognostic genes with a significance of $P[S V] \leq 0.001$. The data were sorted with the five-year survival probability.

C. Relationship between survival probability and variation of gene expression among the population. Shown is the five-year survival probability against the standard deviation (Std) of the correlation coefficients of expression profile (20,531 genes) of each pair of patients for each cancer.

D. The pathways and terms enriched for the genes whose expression was highly correlated to survival time. For each cancer, the top 200 most strongly correlated genes were chosen.

Figure 2 Prognostic genes associated with both survival difference and expression changes in cancer.

A. The 40 prognostic genes identified. Firstly, with criteria of a survival difference $\mathrm{P}[\mathrm{SV}] \leq 10^{-3}$ and fold change of expression $\mathrm{FC}(\mathrm{H} / \mathrm{L}) \geq 2$, and by selecting the top ten genes at most for each cancer type, 236 genes were identified in 29 cancer types. Then the 236 genes were further filtered with stricter criteria of $\mathrm{P}[\mathrm{SV}] \leq 10^{-6}$ and $\mathrm{FC}(\mathrm{H} / \mathrm{L}) \geq 4$. The term "effect" indicates the relationship between gene expression and survival, a downward-pointing triangle means a high expression of the gene corresponds to a poor survival, and an upward-pointing triangle means a high expression to a good survival.

B. Principal component analysis (PCA) for the gene expression of the 40 prognostic genes in 29 cancer types.

C. The genes whose expressions highly correlate with survival. The correlation is calculated as a Pearson correlation coefficient (r).

D. Survival curves for the three prognostic genes. 
Figure 3 Genes with the capacity for both prognosis and diagnosis.

A. The 22 genes that are both prognostic and diagnostic. The 236 prognostic genes were further filtered with two new criteria. One was the fold change of expression between the cancer tissues (C) and the normal tissues (N), namely $\left|\log _{2}(\mathrm{FC}[\mathrm{C} / \mathrm{N}])\right| \geq 2$. The other was the capacity of differentiating cancer and normal cases, which was assessed by AUC of ROC curve, with the criterion of AUC $\geq 0.8$.

B. The gene expression of the 22 genes in normal tissues, and both low- and high-expression cancer groups (Cancer-L and Cancer-H).

C. Demonstration of two genes, CDC20 and MYEOV, in prognosis and diagnosis. Left panels, expression levels of the genes in normal and cancer tissues (Cancer-H and Cancer- $\mathrm{L}$ ). Middle panels, the ROC curves of diagnosis. Right panels, the survival curves of the high- and low-expression groups. The $\mathrm{p}$ value of the log-rank test and the number of the groups are indicated.

Figure 4 Prognostic and diagnostic value of fifteen immunoregulation-related genes.

Shown is the survival difference (P[SV]) between the high- and low-expression groups of each immunoregulation-related gene. The AUC indicates the diagnostic capacity in differentiating cancer tissues.

Figure 5 Association between the expression of the prognostic genes and the somatic mutations.

A. Links between the expression of the prognostic genes and the mutation profile of the top 200 frequently mutated genes. For each prognostic gene, a chi-squared test was employed to test the association between its expression level and the mutation times of the 200 mutated genes in each cancer. The mutated genes with $\mathrm{p} \leq 0.001$ were included in an enrichment test for GO term, KEGG pathway, InterPro domain, and SMART mode. A cutoff of $\mathrm{p} \leq 0.05$ was used to find the enriched terms and pathways. If the criteria were satisfied, a link between the prognostic gene and the mutated pathway (term) was counted once. This was done for each cancer type. The heat map here displays the counts for the links within 29 cancer types.

B. Generalized linear regression of the expression of the prognostic genes with the mutation profile of the top 200 mutated genes. The network displays the regression results after filtering with $\mathrm{p}<0.05$ in the chi-squared test. In the network, the yellow-marked genes are the prognostic genes and the other genes are the mutated genes. A line from the mutated gene to the prognostic gene indicates that the mutation relates to the expression of the prognostic gene. Blue and red lines mean negative and positive effects, respectively. The line width is 
bioRxiv preprint doi: https://doi.org/10.1101/526285; this version posted January 22, 2019. The copyright holder for this preprint (which was not certified by peer review) is the author/funder, who has granted bioRxiv a license to display the preprint in perpetuity. It is made available under aCC-BY-NC-ND 4.0 International license.

proportional to the significance ( $\mathrm{p}$ value of chi-squared test). 

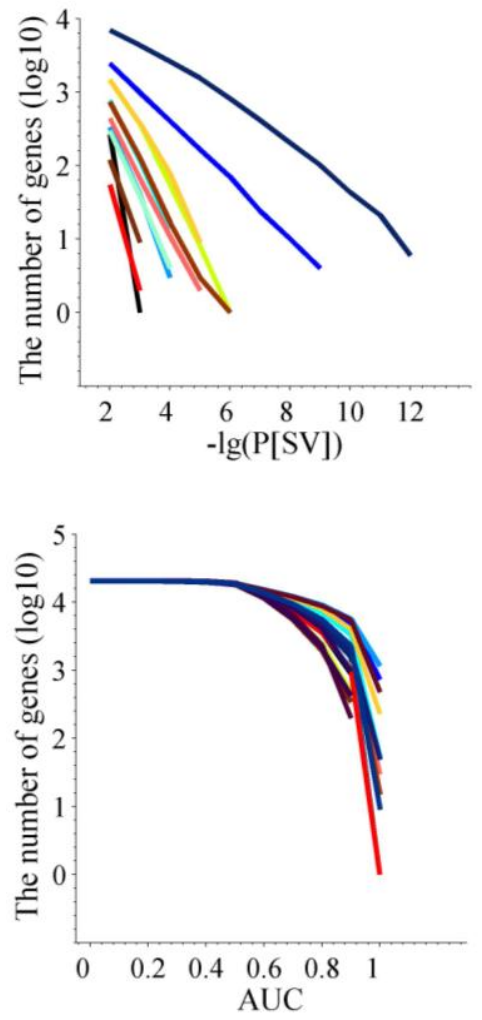

B

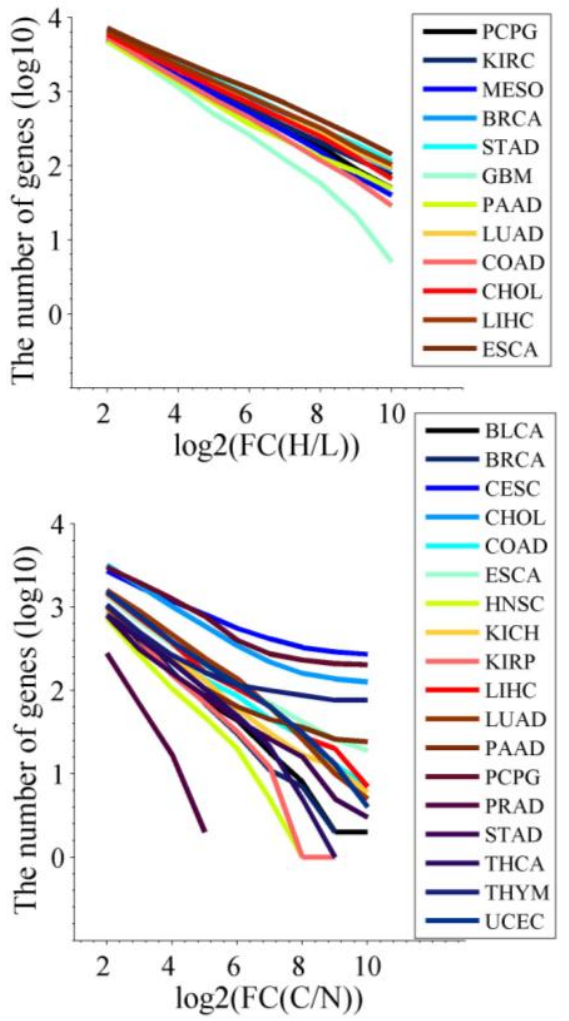

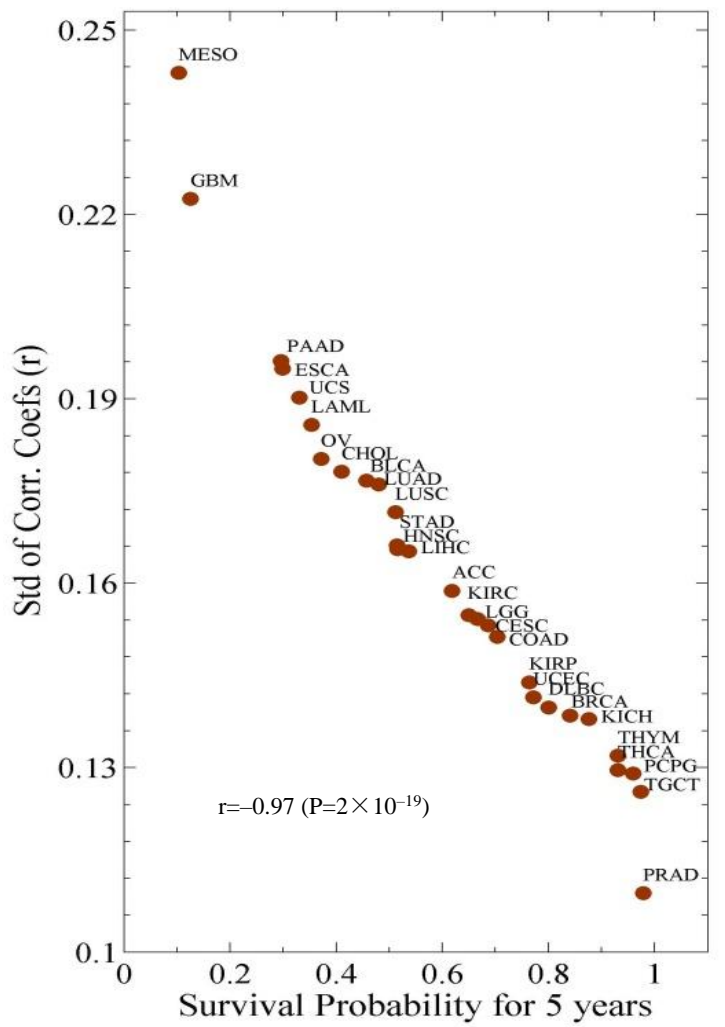

$\mathrm{P}[\mathrm{SV}]<=0.001$

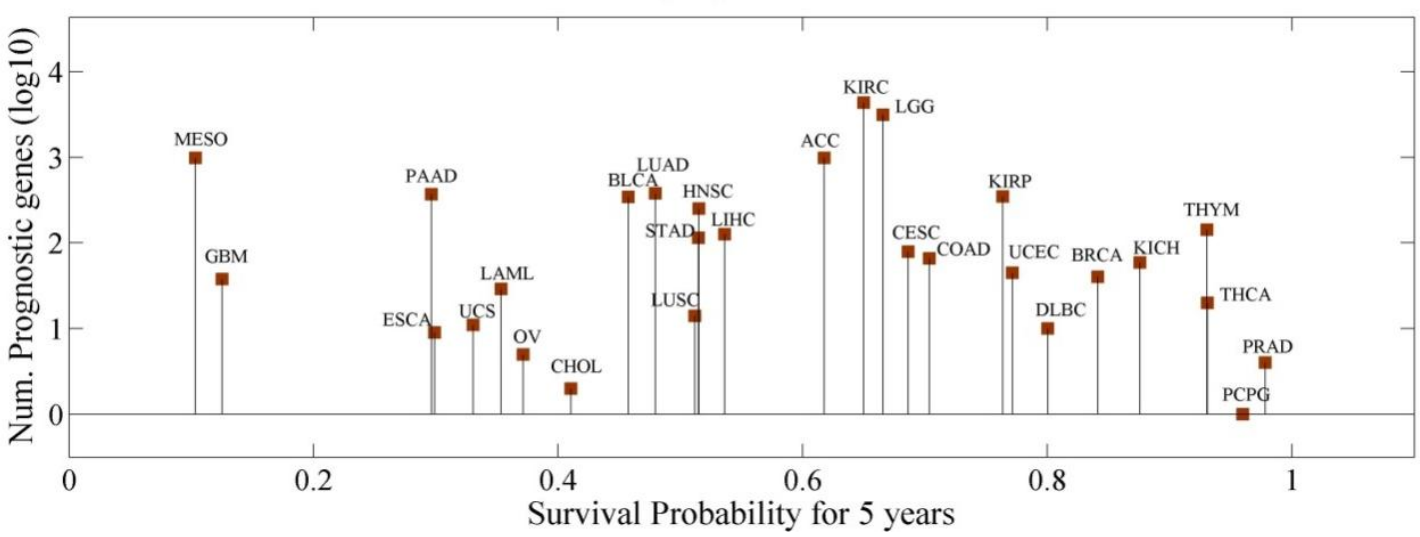

$\mathrm{D}$

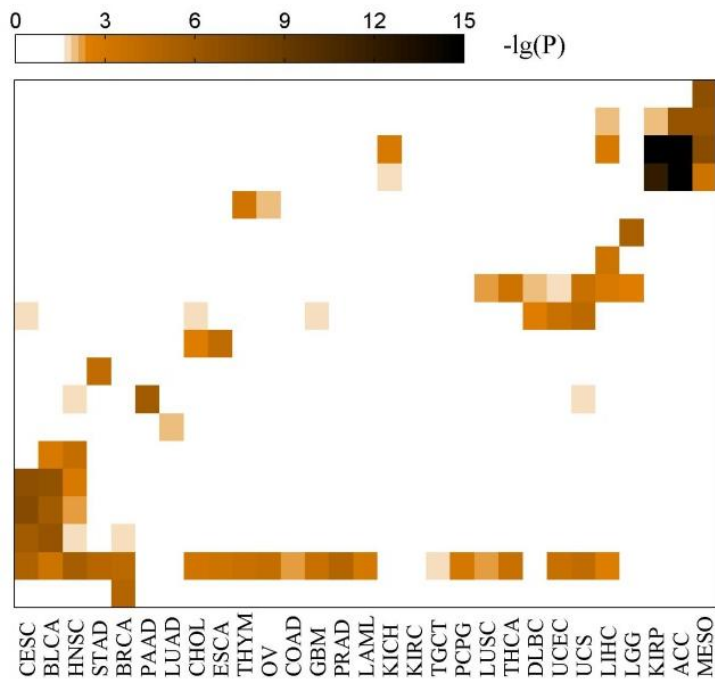

GO:0051607 defense response to virus

GO:0000281 mitotic cytokinesis

Cell cycle

GO:0051301 cell division

hsa04974:Protein digestion and absorption

GO:0048471 perinuclear region of cytoplasm

IPR009030:Insulin-like growth factor binding protein, $\mathrm{N}$-terminal

GO:0005509 calcium ion binding

GO:0007155 cell adhesion

GO:0045926 negative regulation of growth

hsa04020:Calcium signaling pathway

GO:0030054 cell junction

hsa04014:Ras signaling pathway

GO:0050853 $\sim \mathrm{B}$ cell receptor signaling pathway

hsa 04514: Cell adhesion molecules (CAMs)

GO:0031295 T cell costimulation

GO:0006955 immune response

Glycoprotein

GO:0030574 collagen catabolic process 
USP2

UHRF1

SHOX2
SHCBP1

SGOL1

RCAN3

PPFIBP2

OTOF

OSR2

NPTX2

MYBL2

MCM10

MC1R

LOC388387

KLRA1

KIF14

KCNK5

ISL2

IRX5

HOXD10

GTSE1

GSTA1

FAM72D

FAM160A1

FAM159A

ERCC6L

DKK 1

CILP

CEP55

CDK1

CDCA8

CDC20

C7orf29

C5orf32

C1QTNF6

ATP6V1C2

ASPM

ANKRD56

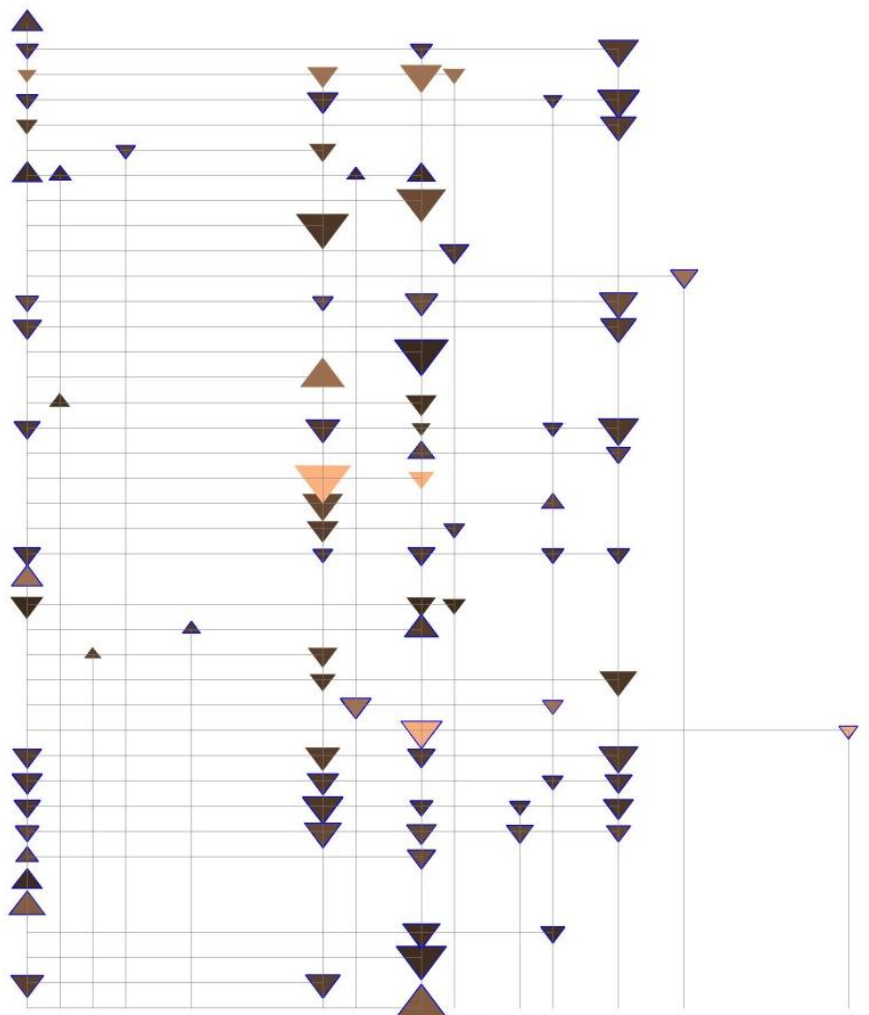

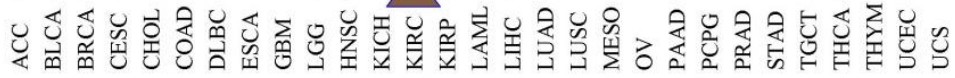

$-\lg (\mathrm{P}[\mathrm{SV}])$

$\log 2(\mathrm{FC}(\mathrm{H} / \mathrm{L})){ }_{2}^{2} \Delta_{4}^{3} \Delta_{4}^{4}{ }_{6}^{5}{ }_{8}^{6} \Delta_{8}^{7}$

Effect

$\triangle_{\text {Pretective }}^{\nabla} \quad \nabla_{\text {Hazard }}$

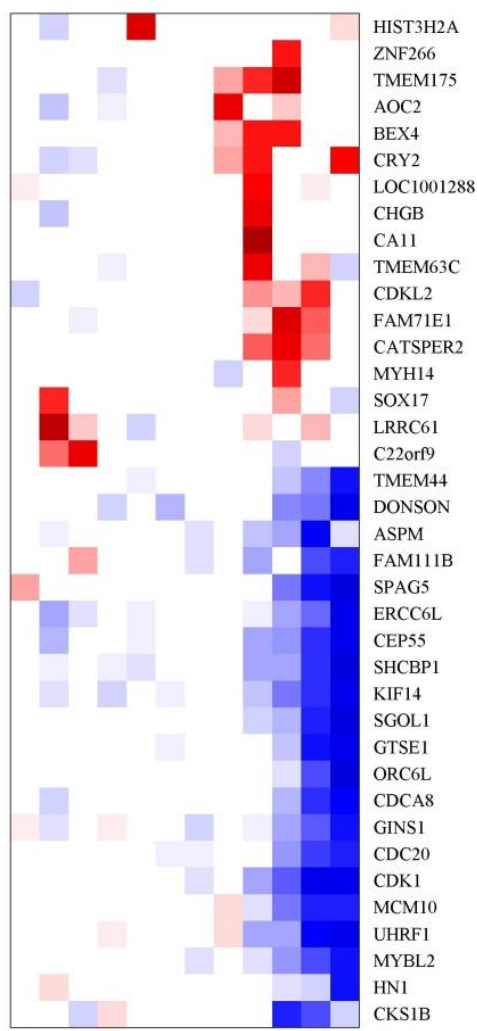

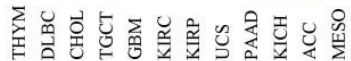

B



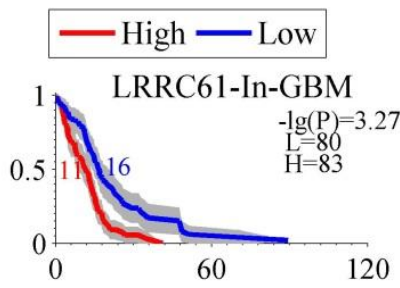
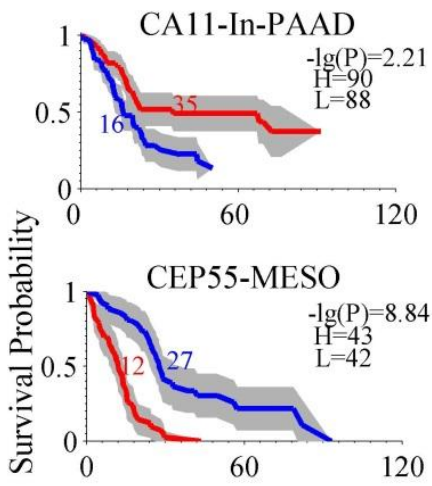

Time (months) 


\section{6 genes $\longrightarrow|\log 2 \mathrm{FC}(\mathrm{C} / \mathrm{N})|>2 \longrightarrow 22$ genes AUC $>0.8$}

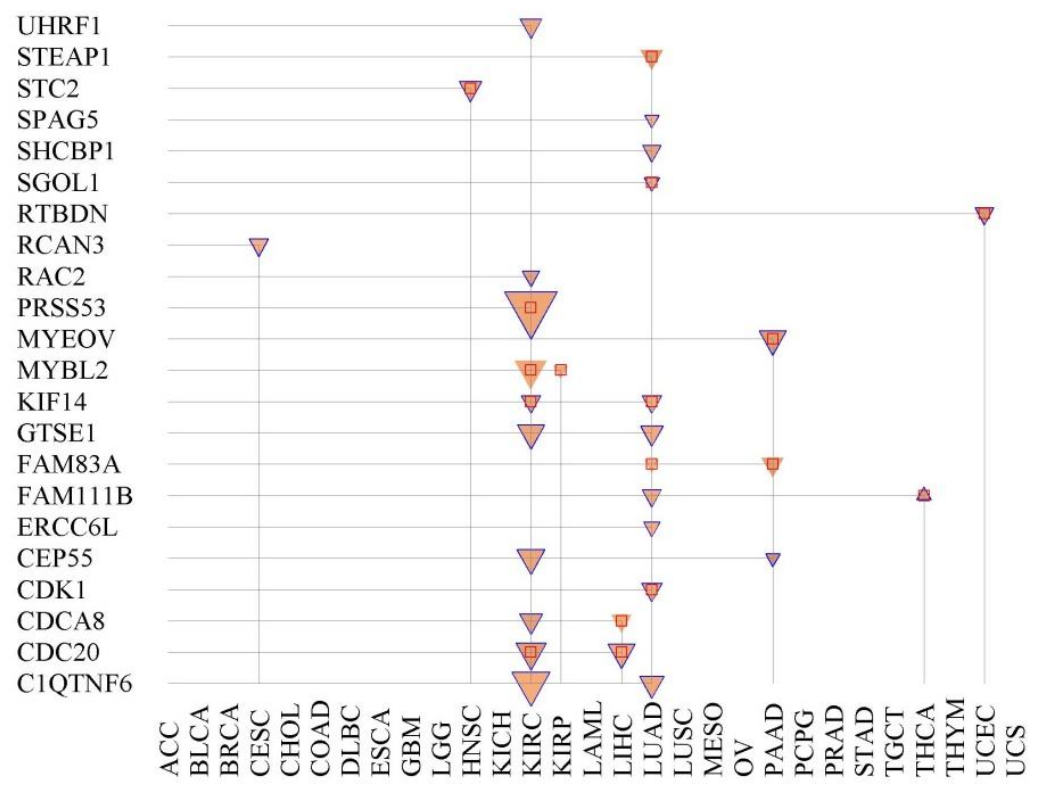

Effect

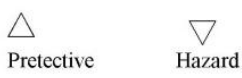

B

UHRF1-KIRC STEAP1-LUAD STC2-HNSC SPAG5-LUAD SHCBP1-LUAD SGOL1-LUAD RTBDN-UCEC RCAN3-CESC RAC2-KIRC PRSS53-KIRC MYEOV-PAAD MYBL2-KIRP MYBL2-KIRC KIF14-LUAD GTSE1-LUAD GTSE1-KIRC FAM83A-PAAD FAM83A-LUAD FAMI11B-LUAD ERCC6L-LUAD CEP55-KIRC - CDK1-LUAD CDCA8-LIHC CDC20-LIHC A C1QTNF6-LUAD CIQTNF6-KIRC $\lg (\mathrm{FPKM}+1) \triangle \triangle \triangle$ $\begin{array}{llll}0 & 1 & 2 & 3\end{array}$

\begin{abstract}
AUC
\end{abstract}
$\mathrm{FC}(\mathrm{H} / \mathrm{L})>4$
C
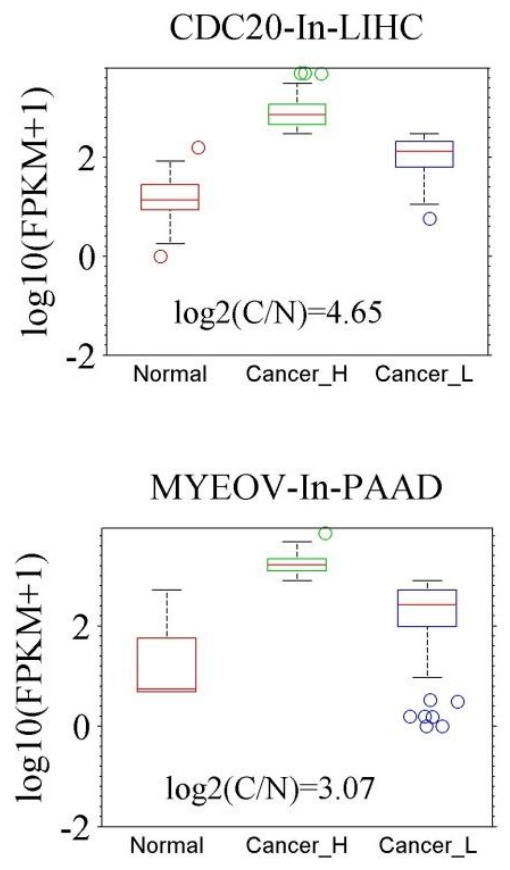

Cell types

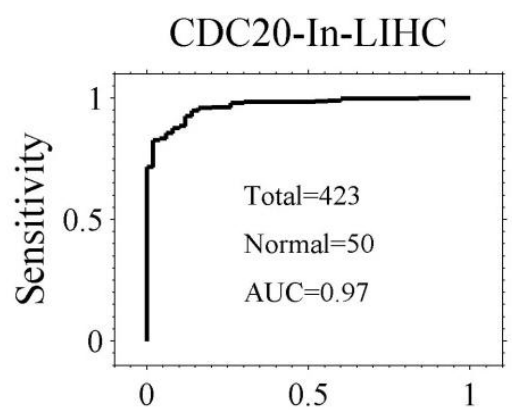

MYEOV-In-PAAD

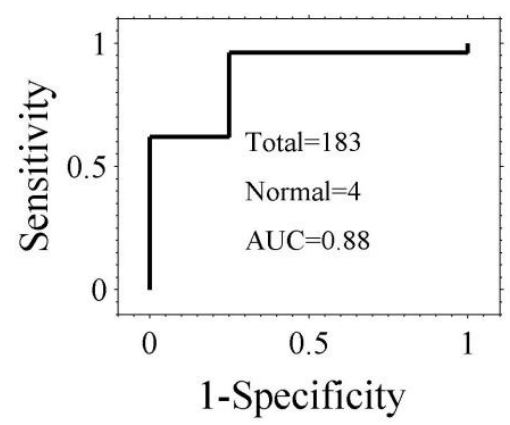

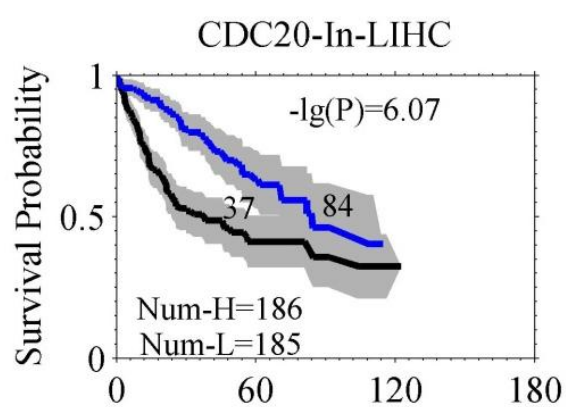

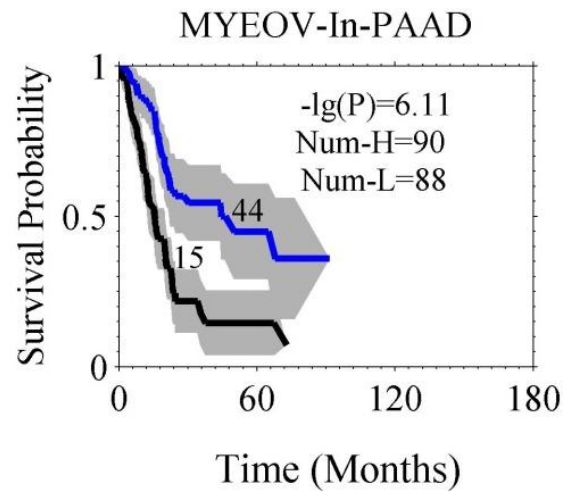



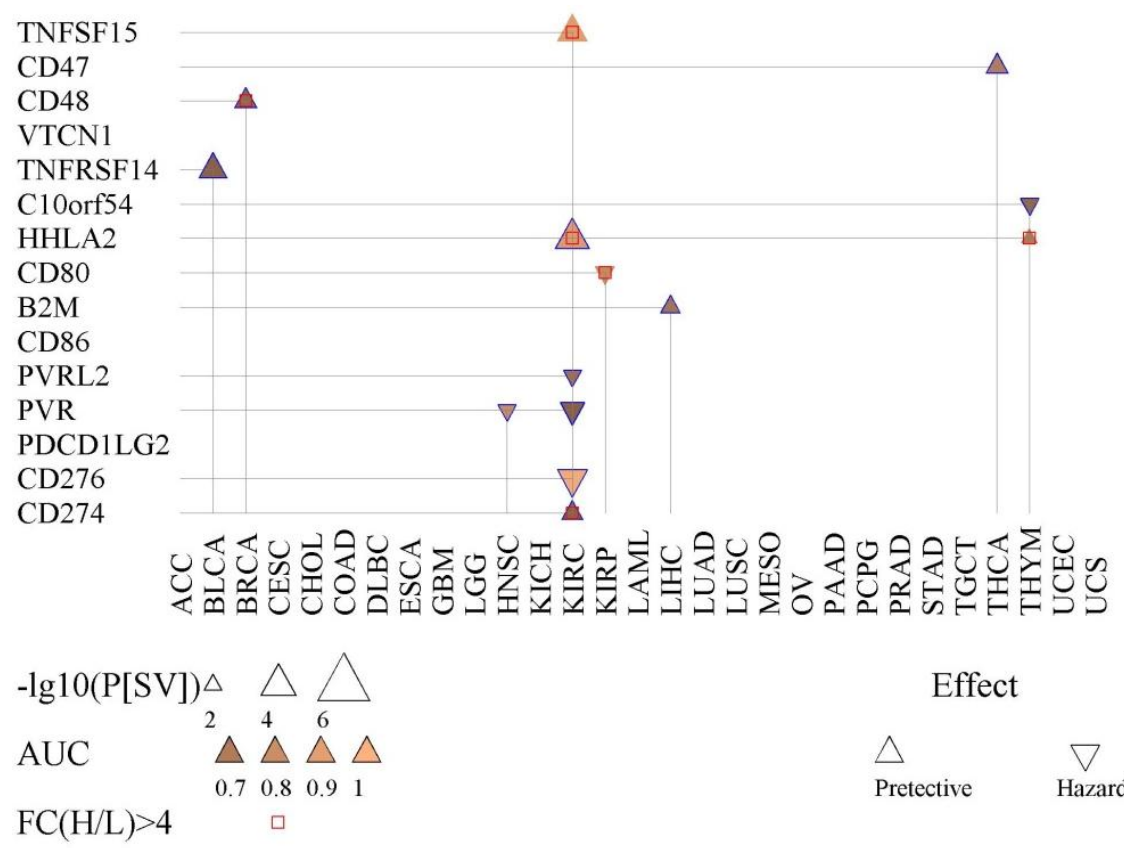

Effect

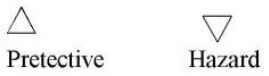




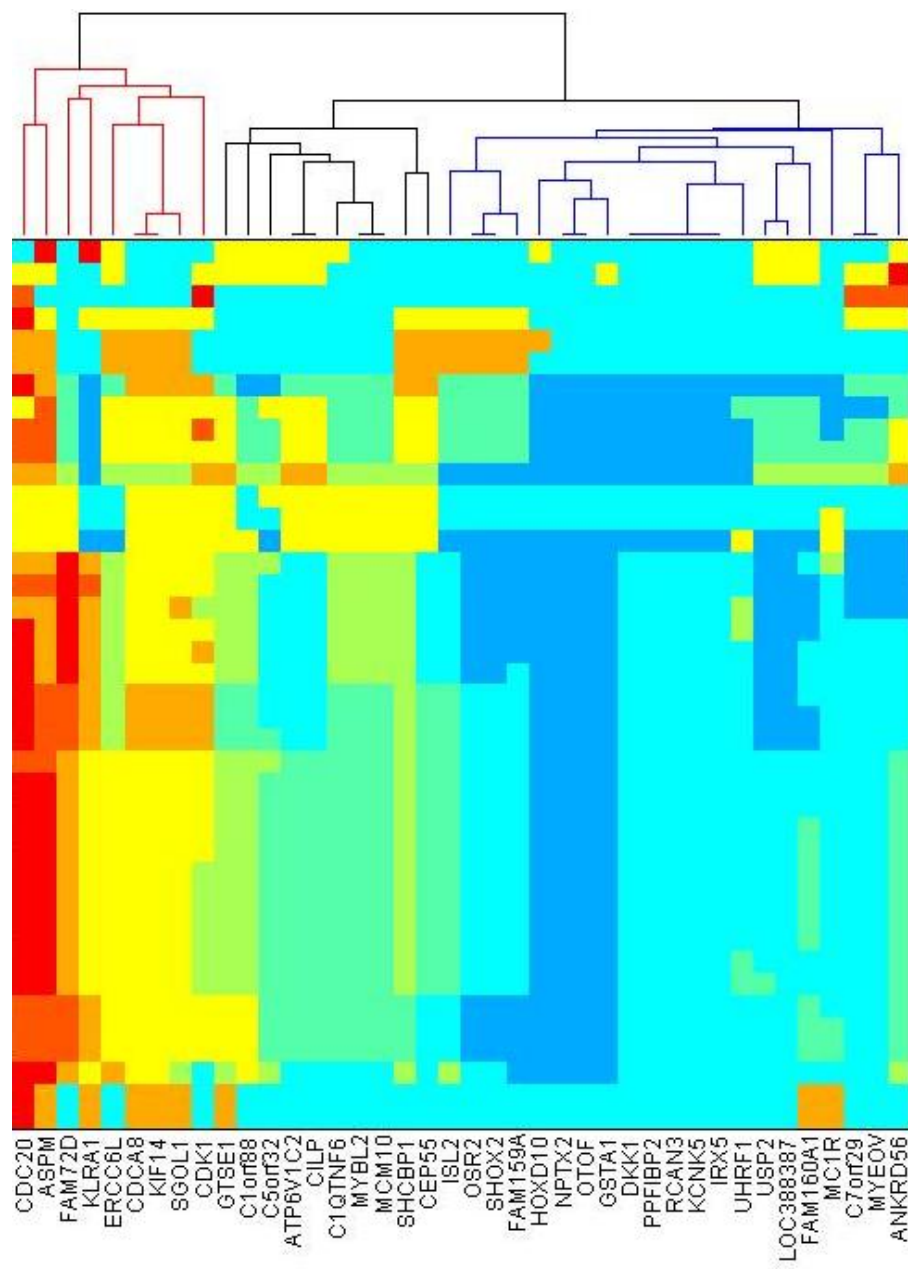

GO:0005509 calcium ion binding hsa04720: Long-term potentiation hsa04530:Tight junction

hsa0 4320:Dorso-ventral axis formation

PR009030:Insulin-like growth factor binding protein, N-terminal hsa04020:Calcium signaling pathway

hsa04921: Oxytocin signaling pathway

hsa04510:Focal adhesion

hsa04068:Foxo signaling pathway

hsa0 4012: ErbB signaling pathway

hsa05211:Renal cell carcinoma

hsa05146:Amoebiasis

hsa04973:Carbohydrate digestion and absorption domain:PI3KIPI4K

hsa05222:Small cell lung cancer

Tumor suppressor

hsa05016: Huntington's disease

hsa05166: HTLV-l infection

hsa05161: Hepatitis B

hsa04919:Thyroid hormone signaling pathway

hsa05230: Central carbon metabolism in cancer

hsa05206:MicroRNAs in cancer

hsa04151:PI3K-Akt signaling pathway

hsa05200:Pathways in cancer

hsa05215: Prostate cancer

hsa05213: Endometrial cancer

hsa05214:Glioma

hsa05218: Melanoma

hsa05223:Non-small cell lung cancer

hsa05219: Bladder cancer

hsa05160:Hepatitis C

hsa05212:Pancreatic cancer

hsa04010:MAPK signaling pathway

hsa 05205:Proteoglycans in cancer

hsa05210:Colorectal cancer

hsa05216: Thyroid cancer

hsa05220:Chronic myeloid leukemia

Methylation

isa05414:Dilated cardiomyopathy

hsa05410:Hypertrophic cardiomyopathy ( $\mathrm{HCM}$ )

뚠ㅁำ

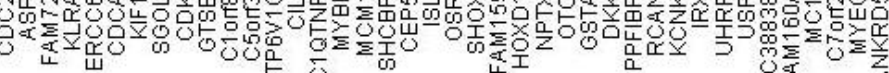

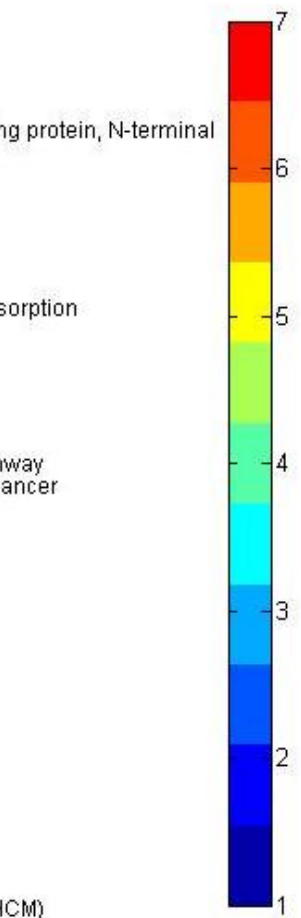

B
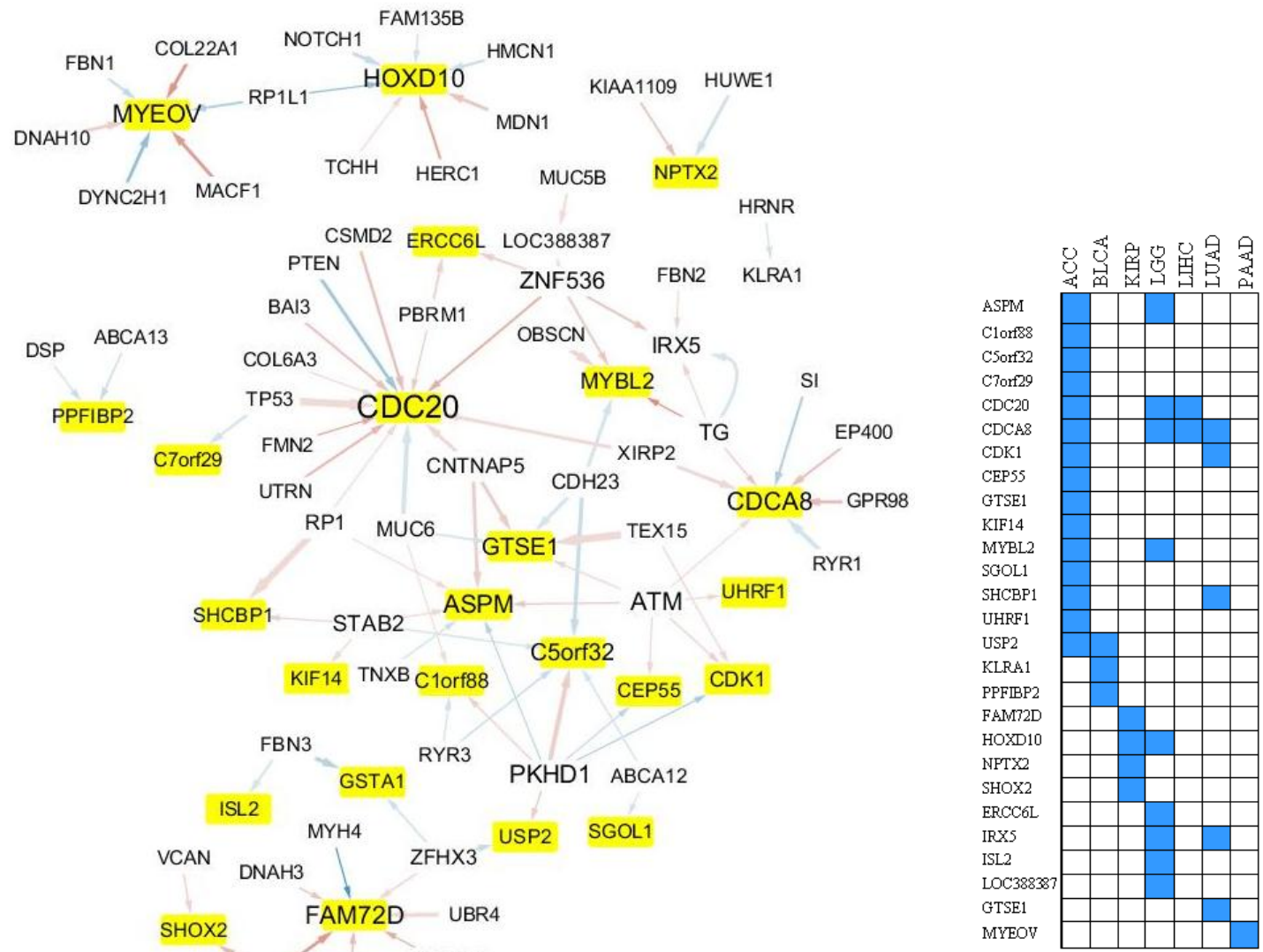

SHOX2

USP34 PKD1L1

Number of Cancers $-\lg (\mathrm{P})>2$

Source of the network 


\title{
Supplementary Information
}

\section{Comprehensive identification of survival-associated genes for cancers}

\author{
Hongde Liu ${ }^{1 *}$, Kun $\mathrm{Luo}^{2}$, Huamei $\mathrm{Li}^{1}, \mathrm{Xiao} \mathrm{Sun}^{1}$
}

${ }^{1}$ State Key Laboratory of Bioelectronics, School of Biological Science \& Medical

Engineering, Southeast University, Nanjing 210096, China

${ }^{2}$ Department of Neurosurgery, Xinjiang Evidence-Based Medicine Research Institute, First Affiliated Hospital of Xinjiang Medical University, Urumqi 830054, China

\section{*The corresponding author:}

Hongde Liu, e-mail: liuhongde@ seu.edu.cn (HDL).

\section{Authors' e-mails:}

Hongde Liu: liuhongde@seu.edu.cn (HDL);

Kun Luo: luokun_2822@sohu.com (KL);

Huamei Li: li_hua_mei@163.com(HML);

Xiao Sun: xsun@seu.edu.cn(XS). 


\section{Materials and methods}

\section{Datasets}

Gene expression data, survival data, and mutation data used in this study were retrieved from TCGA project from the initial release of Genomic Data Commons (GDC) in October 2016 (https://portal.gdc.cancer.gov/) using RTCGAToolbox. ${ }^{1}$ A total of 9523 samples across 29 tumor types were downloaded, including 8811 tumor tissues and 712 non-tumor tissues. Microarray-based gene expression data (gene expression omnibus ID: GSE21501 for pancreatic cancer ${ }^{2}$ ) were retrieved for validating the expression markers for survival.

\section{Identification of the prognostic genes}

Genes whose expression is associated with a differential overall survival were identified with a log-rank test in a Kaplan-Meier survival model. In each cancer type, for each gene, patients were classified into two groups using the expression median of the gene as a cutoff. The two groups were named the high-expression group $(\mathrm{H})$ and the low-expression group (L), depending on whether the expression level was higher or lower than the median, respectively. The survival difference was tested in the two groups. In identifying, we considered both survival difference and the expression change between the two groups. First, the criteria of a survival difference $\mathrm{P}[\mathrm{SV}] \leq 10^{-3}$ and a fold change of expression $(\mathrm{FC}(\mathrm{H} / \mathrm{L})) \geq 2$ were applied to 20,531 genes for 29 cancer types, resulting in a list of 236 genes by choosing the top ten genes. Then, stricter criteria, $\mathrm{P}[\mathrm{SV}] \leq 10^{-6}$ and $\mathrm{FC}[\mathrm{H} / \mathrm{L}]>4$ were applied to the 236 genes, resulting in a list of 40 genes. Finally, we were interested in the possibility of the prognostic genes acting as markers to diagnose cancer and normal tissues. Thus, the expression difference between cancer $(\mathrm{C})$ and control $(\mathrm{N})$ tissues was tested. The area under the curve (AUC) of a receiver operating characteristic (ROC) curve and the expression fold change $(\mathrm{FC}(\mathrm{C} / \mathrm{N}))$ between the cancer and normal tissues were employed to indicate the difference, namely $\mathrm{AUC} \geq 0.8$ and $\left|\log _{2}[\mathrm{FC}(\mathrm{C} / \mathrm{N})]\right| \geq 2$, resulting in a list of 22 genes.

We also identified prognostic genes another way, by examining the Pearson correlation coefficient between gene expression and survival time among the population. Candidate genes were those with a large positive or negative correlation.

\section{Analysis of association between immunoregulation-related gene expression and survival time}

Fifteen immunoregulation-related genes were considered, including $P D L-1, P D L-2$, and $P V R$. PDL-1 and PDL-2 are ligands of programmed death-1 (PD-1), which is involved in the inhibition of T cells, ${ }^{3}$ and PVR is a ligand of TIGIT. The binding of the two inhibits NK cell 
cytotoxicity. $^{4}$

\section{Analysis of the mutations in cancers}

For each gene, mutations were counted in all cancers. A mutation rate (frequency) of the gene is the ratio of the total mutations in the gene to the number of all patients in all cancers. The top 200 frequently mutated genes were used in a regression model for the expression of the prognostic genes to examine what kind of mutations contributed to the expression levels of the prognostic genes (see below). Given that the mutation count is related to both gene length and gene expression, ${ }^{5,6}$ the mutation rate was normalized by dividing the rate by gene length [Kbp].

We investigated the relationship between the mutation counts and the survival time. The survival time was truncated at a survival probability of $60 \%$. We also tested the relationship between the normalized mutation rate and the expression level.

\section{Regression for the expression of the prognostic genes with the mutation counts}

The 40 prognostic genes, which were identified with $\mathrm{P}[\mathrm{SV}] \leq 10^{-6}$ and $\mathrm{FC}[\mathrm{H} / \mathrm{L}] \geq 4$, and the top 200 frequently mutated genes, were included in this section of analysis. Firstly, the prognostic genes were aligned to the pathways enriched for the mutated genes and GO terms. For each prognostic gene, the dependence between its expression and the mutation counts of the 200 mutated genes were tested with a chi-squared $\left(\chi^{2}\right)$ test for each cancer. The mutated genes with $\mathrm{p} \leq 0.001$ ( $\chi^{2}$ test) were included in an enrichment analysis on GO terms, KEGG pathways, InterPro domains, and SMART modes, based on a hypergeometric distribution or Fisher's exact test. A cutoff of $\mathrm{p} \leq 0.05$ was used to find the enriched terms and pathways. Upon satisfaction of those criteria, a link between the prognostic gene and the mutated pathway (terms) was counted. This was done for all cancers to see how many cancer types shared the link.

Secondly, we carried out a generalized linear regression of the expression of the prognostic gene with the mutation counts of the top 200 frequently mutated genes for each cancer type. The regression generated a set of parameters indicating the contribution of the mutation in explaining the expression level of the prognostic gene. Only mutated genes with a significant parameter were used to construct a network.

\section{Enrichment analysis}

The enrichment analysis for the 236 prognostic genes and the top 200 frequently mutated genes was carried out with DAVID. ${ }^{7}$

\section{Other analysis in the work}


The activation status of pathways was assessed with SPIA. ${ }^{8}$ Survival analysis and the survival comparison were carried out by an inhouse Matlab program (Suppl-4).

\section{Abbreviation for cancer type}

LAML, Acute Myeloid Leukemia;

ACC, Adrenocortical carcinoma;

BLCA, Bladder Urothelial Carcinoma;

LGG, Brain Lower Grade Glioma;

BRCA, Breast invasive carcinoma;

CESC, Cervical squamous cell carcinoma and endocervical adenocarcinoma;

CHOL, Cholangiocarcinoma;

LCML, Chronic Myelogenous Leukemia;

COAD, Colon adenocarcinoma;

ESCA, Esophageal carcinoma;

GBM, Glioblastoma multiforme;

HNSC, Head and Neck squamous cell carcinoma;

KICH, Kidney Chromophobe;

KIRC, Kidney renal clear cell carcinoma;

KIRP, Kidney renal papillary cell carcinoma;

LIHC, Liver hepatocellular carcinoma;

LUAD, Lung adenocarcinoma;

LUSC, Lung squamous cell carcinoma;

DLBC, Lymphoid Neoplasm Diffuse Large B-cell Lymphoma;

MESO, Mesothelioma;

OV, Ovarian serous cystadenocarcinoma;

PAAD, Pancreatic adenocarcinoma;

PCPG, Pheochromocytoma and Paraganglioma;

PRAD, Prostate adenocarcinoma;

STAD, Stomach adenocarcinoma;

TGCT, Testicular Germ Cell Tumors;

THYM, Thymoma;

THCA, Thyroid carcinoma;

UCS, Uterine Carcinosarcoma;

UCEC, Uterine Corpus Endometrial Carcinoma. 


\section{References}

1 Samur, M. K. RTCGAToolbox: a new tool for exporting TCGA Firehose data. PloS one 9, e106397, doi:10.1371/journal.pone.0106397 (2014).

2 Stratford, J. K. et al. A six-gene signature predicts survival of patients with localized pancreatic ductal adenocarcinoma. PLoS medicine 7, e1000307, doi:10.1371/journal.pmed.1000307 (2010).

3 Xu-Monette, Z. Y., Zhou, J. \& Young, K. H. PD-1 expression and clinical PD-1 blockade in B-cell lymphomas. Blood 131, 68-83, doi:10.1182/blood-2017-07-740993 (2018).

4 Stanietsky, N. et al. The interaction of TIGIT with PVR and PVRL2 inhibits human NK cell cytotoxicity. Proceedings of the National Academy of Sciences of the United States of America 106, 17858-17863, doi:10.1073/pnas.0903474106 (2009).

5 Bailey, M. H. et al. Comprehensive Characterization of Cancer Driver Genes and Mutations. Cell 174, 1034-1035, doi:10.1016/j.cell.2018.07.034 (2018).

6 Lawrence, M. S. et al. Mutational heterogeneity in cancer and the search for new cancer-associated genes. Nature 499, 214-218, doi:10.1038/nature12213 (2013).

7 Huang da, W., Sherman, B. T. \& Lempicki, R. A. Systematic and integrative analysis of large gene lists using DAVID bioinformatics resources. Nature protocols 4, 44-57, doi:10.1038/nprot.2008.211 (2009).

8 Tarca, A. L. et al. A novel signaling pathway impact analysis. Bioinformatics 25, 75-82, doi:10.1093/bioinformatics/btn577 (2009). 


\section{Figure Legend}

Figure S1 Cancers exhibit a great difference in the number of the genes that are associated with survival difference $\mathrm{P}[\mathrm{SV}]$ and have a diagnostic value (AUC).

A. Gene numbers at different cutoffs of both survival difference and differential expression between cancer and control. For each gene in each cancer, P[SV] was calculated with a log-rank test in the high- and low-expression groups that were obtained by dividing the cancer tissues by the median expression of the gene. AUC is the area under the ROC curves in differentiating the cancer and the control with the gene expression.

B. The pathways that are significantly enriched for the genes whose expression changes greatly among cancer tissues $(\mathrm{FC}(\mathrm{H} / \mathrm{L}) \geq 2)$. The analysis was done with the SPIA tool.

Figure S2 Forty prognostic genes identified by the criteria $\mathrm{P}[\mathrm{SV}] \leq 10^{-6}$ and $\mathrm{FC}[\mathrm{H} / \mathrm{L}] \geq 4$.

A. The identification flow chart. $\mathrm{P}[\mathrm{SV}]$ is from the log-rank test in the high- and low-expression groups that were obtained by dividing the cancer tissues by the gene's median expression.

B. Venn diagram showing the number of the overlapping genes in our study (A) and in the literature (B) (Uhlen M. et al. 2017, Science 357). The overlapping genes are DKK1, EMP1, EPS8, FAM83A, MET, MGAT4A, MYEOV, and PGK1.

C. Subcellular location of the forty prognostic genes.

D. An enrichment analysis for the identified 236 and 40 prognostic genes with the DAVID tool for KEGG pathways and GO terms.

E. The survival curves of the MYEOV high- and low-expression cancer groups in PAAD using microarray data (GEO ID, GSE21501).

Figure S3 Sample to demonstrate that a prognostic gene is not necessarily suitable for discriminating cancer tissues from normal tissues.

A. The survival curves of the $D K K 1$ high- and low-expression cancer groups.

B. Boxplot of the expression of $D K K 1$ in LUAD tissues and normal tissues. LUAD tissues are divided into high- and low-expression groups based on the median expression.

C. Expression of $D K K 1$ in each LUAD tissue.

D. Average expression of $D K K 1$ in 21 cancers and controls.

Figure S4 Survival curves and ROC curves to show the capacity of the genes in both prognosis and diagnosis in specific cancers.

A. The survival curves of the high- and low-expression groups. The difference significance $\mathrm{P}[\mathrm{SV}]$ and the median survival time are indicated. 
B. ROC curves of diagnosis.

Figure S5 Characteristics of $C D C 20$ in prognosis and diagnosis in 21 cancers.

A. The boxplots indicate gene expression of $C D C 20$ in normal tissues, and the high- and low-cancer groups.

B. Plot of the prognostic and diagnostic values of $C D C 20$ in cancers.

Figure S6 Most frequently mutated genes in cancers.

A. Shown are the top 40 mutated genes for all cancer types. The vertical axis represents the mutation rate, namely a rate of total number of mutations occurring in the gene to the number of all patients in all cancer types.

B. An enrichment analysis for the top 200 frequently mutated genes.

C. Survival time of the patients with the mutations in each gene. The survival time is truncated at a survival probability of $60 \%$; each patient that has a mutation in the gene was counted. Shown is a heat map indicating the distribution of the survival time against the mutation counts. Lightness is proportional to the number of genes.

D. Relationship between the expression level and the mutation rate for each gene. The mutation rate was normalized by dividing the rate by gene length [Kbp]. The expression data (FPKM) were normalized into percentile in each sample (patient). For each gene, its expression percentile was plotted to its mutation rate per base pair.

\section{Figure S7}

A. Top 40 mutated genes for all cancer types. The mutation rate was normalized by dividing by gene length [Kbp].

B. Venn diagram showing the overlapping genes between the top 200 genes ranked by the maximum mutation rate before and after the normalization by gene length.

C. Distribution of the normalized mutation rate.

D. An enrichment analysis for the top 200 genes ranked by the normalized maximum mutation rate.

E. An enrichment analysis for the top 200 genes whose mutations associate to the shortest survivals (from Fig. S6C). 


\section{Supplementary Files}

\section{Suppl-1}

The prognostic genes identified at criteria of $\mathrm{P}[\mathrm{SV}] \leq 10^{-3}$ and $\mathrm{FC}(\mathrm{H} / \mathrm{L}) \geq 2$, selecting the top ten genes for each cancer type. There were a total of 236 genes in 29 cancer types. Here, in each cancer type, for each gene, patients are classified into high $(\mathrm{H})$ and low $(\mathrm{L})$ expression groups using the expression median of the gene as a cutoff. The survival difference (P[SV]) was tested between the two groups with a log-rank test. $\mathrm{FC}(\mathrm{H} / \mathrm{L})$ means the fold change of average expression between the two groups. In the files, chi-square is the value of $\chi^{2}$ in the log-rank test, T-middle- $\mathrm{H}$ and T-middle- $\mathrm{L}$ are the time at $50 \%$ survival probability for the high- and low-expression groups, respectively, and mean-H and mean-L are the mean expression in the high- and low-expression groups, respectively. The gene symbol and cell type are the official symbol of the prognostic gene and the corresponding cancer type, respectively.

\section{Suppl-2}

The prognostic genes identified at criteria of $\mathrm{P}[\mathrm{SV}] \leq 10^{-6}$ and $\mathrm{FC}(\mathrm{H} / \mathrm{L}) \geq 4$. In the file, the term "Effective" indicates the relationship between gene expression and survival, a downward-pointing triangle means a high expression of the gene corresponds to poor survival, and an upward-pointing triangle means a high expression of the gene corresponds to good survival. The other settings are the same as in the Suppl-1 file.

\section{Suppl-3}

The genes that have both prognostic and diagnostic values, with criterion of $\mathrm{P}[\mathrm{SV}] \leq 10^{-4}$, indicating the survival difference between the high and low expression groups, and AUC $\geq 4$, indicating the capacity of the diagnosing cancer. The term $\log _{2}(\mathrm{FC}(\mathrm{C} / \mathrm{N}))$ is the fold change of average expression between the cancer $(\mathrm{C})$ and the control $(\mathrm{N})$. Other settings are same as in Suppl-1 file.

\section{Suppl-4}

The programs (in Matlab) that used in the analysis;

"Survival_Analysis_OKAY.m”, for survival analysis based on Kaplan-Meier survival model. "SR_chi_test_bigdata.m", for survival difference test, namely log-rank test.

“plot_Survival_Anlysis", for plotting the survival curves. 

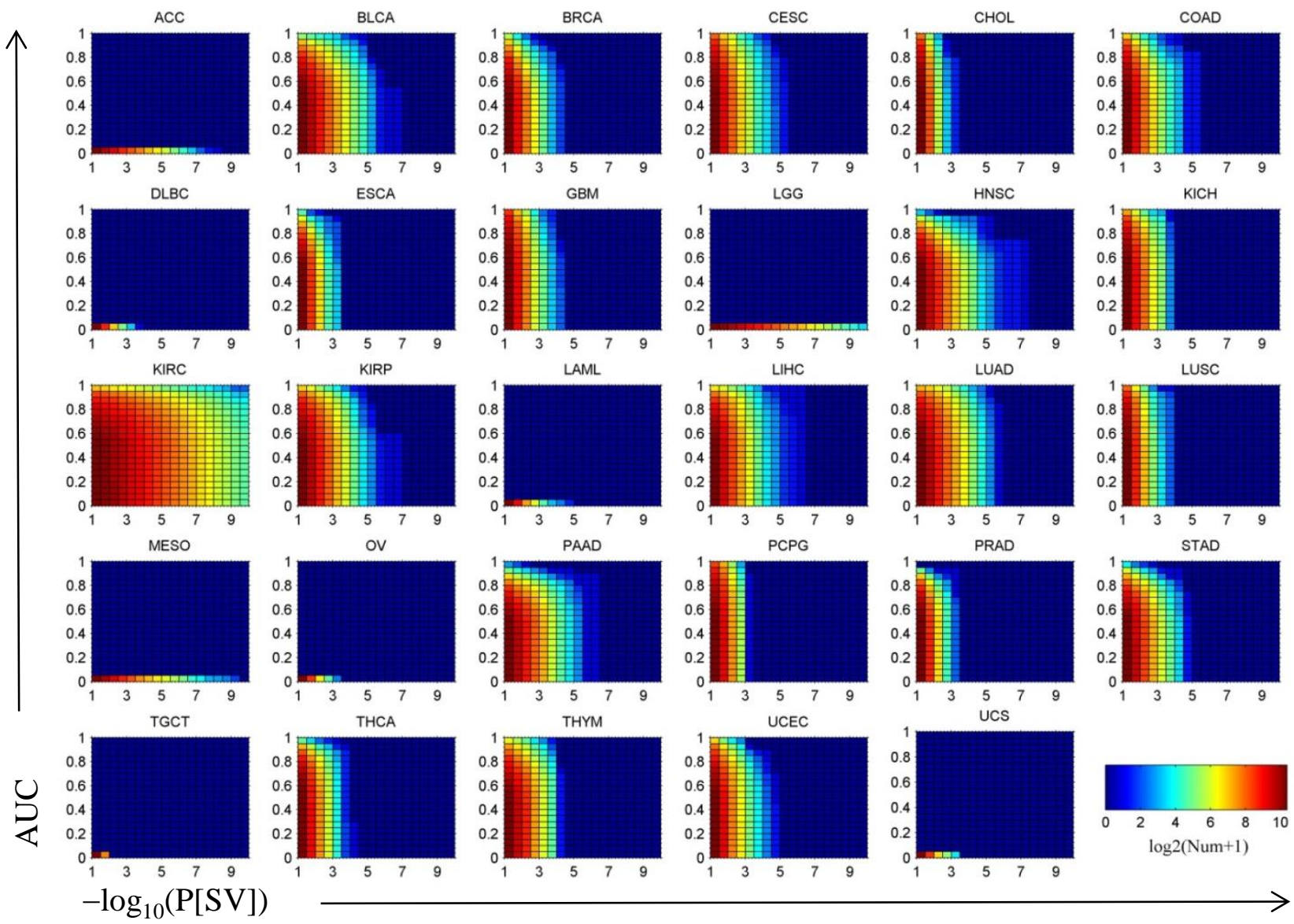

B

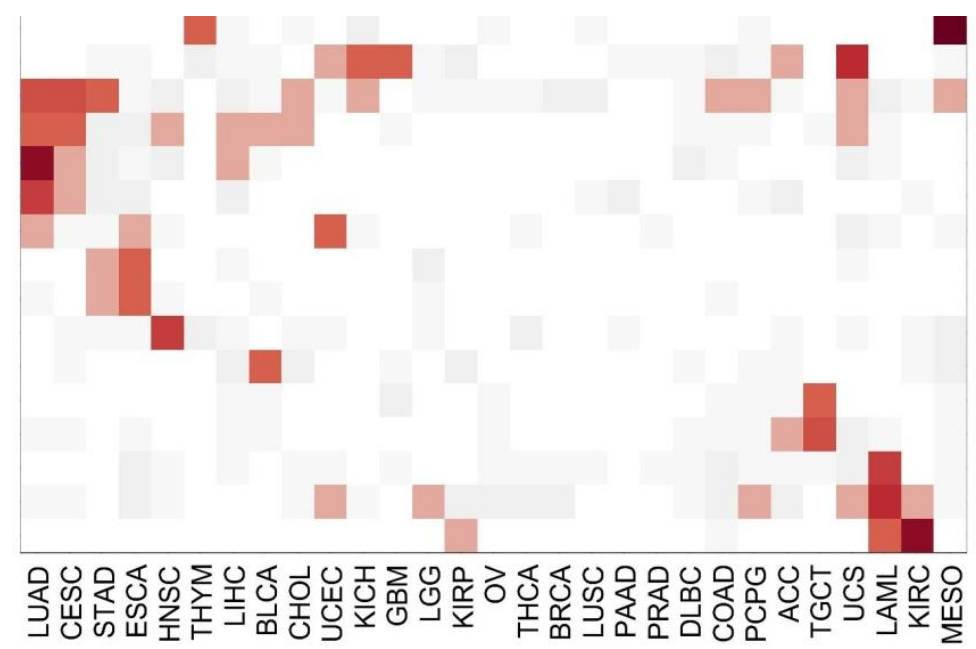

Phototransduction

Rap1 signaling pathway

Renin secretion

Gastric acid secretion

Cocaine addiction

Relaxin signaling pathway

Apelin signaling pathway

PPIER

Neurotrophin signaling pathway

IL-17 signaling pathway

ARSPIDC

Basal cell carcinoma

Melanogenesis

Long-term potentiation

GnRH signaling pathway

ECM-receptor interaction

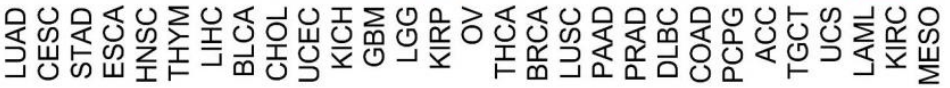

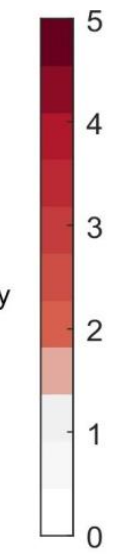

$-\lg (\mathrm{pG})$

Abbr.:

ARSPIDC, AGE-RAGE signaling pathway in diabetic complications;

PPIER, Protein processing in endoplasmic reticulum 


\begin{tabular}{|l}
20531 genes \\
(Total) \\
$\downarrow$ \\
$\mathrm{P}[\mathrm{SV}] \leq 10^{-3} ;$ \\
$\mathrm{FC}(\mathrm{H} / \mathrm{L}) \geq 2 ;$ \\
$\mathrm{Top} 10$ \\
$\downarrow$ \\
236 genes \\
$\downarrow$ \\
$\mathrm{P}[\mathrm{SV}] \leq 10^{-6} ;$ \\
$\mathrm{FC}(\mathrm{H} / \mathrm{L}) \geq 4 ;$ \\
$\downarrow$ \\
40 genes
\end{tabular}

D

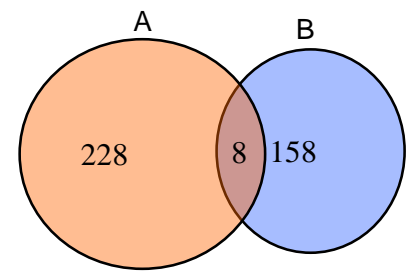

A. Top 20 genes with both $\mathrm{P}[\mathrm{SV}] \leq 10^{-3}$ and $\mathrm{FC}(\mathrm{H} / \mathrm{L}) \geq 2$ in 29 Cancers

B. Top 20 genes with $\mathrm{P}[\mathrm{SV}] \leq 10^{-3}$ in 17 cancers in literature

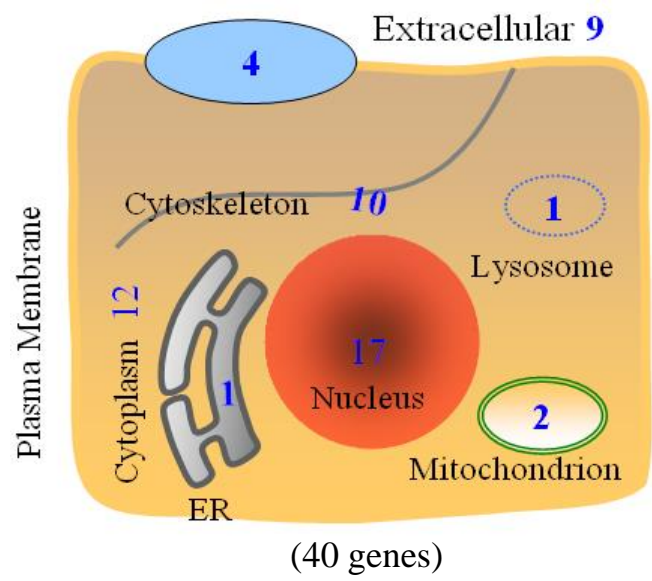

$E$

$\mathrm{T}=236$

GO:0008360 regulation of cell shape $\left(\mathrm{fdr}=10^{0}\right)$

GO:0051301 cell division $\left(\mathrm{fdr}=10^{0.7}\right)$ (7)

GO:0008283 cell proliferation $\left(\mathrm{fdr}=10^{0.7}\right)$

$\begin{array}{lllllllll}0 & 0.5 & 1 & 1.5 & 2 & 2.5 & 3 & 3.5 & 4\end{array}$
$-\log _{10}(\mathrm{p}$-value $)$

$\mathrm{T}=40$

Cell cycle $\left(\mathrm{fdr}=10^{-1.2}\right)$

Cell division $\left(\mathrm{fdr}=10^{-1.2}\right)$

(6)

GO:0007067 mitotic nuclear division $\left(\mathrm{fdr}=(6) 0^{-0.4}\right)$

Cytoskeleton $\left(\mathrm{fdr}=10^{0}\right)$

(5)

MYEOV-PAAD (Microarray data, GSE21501)

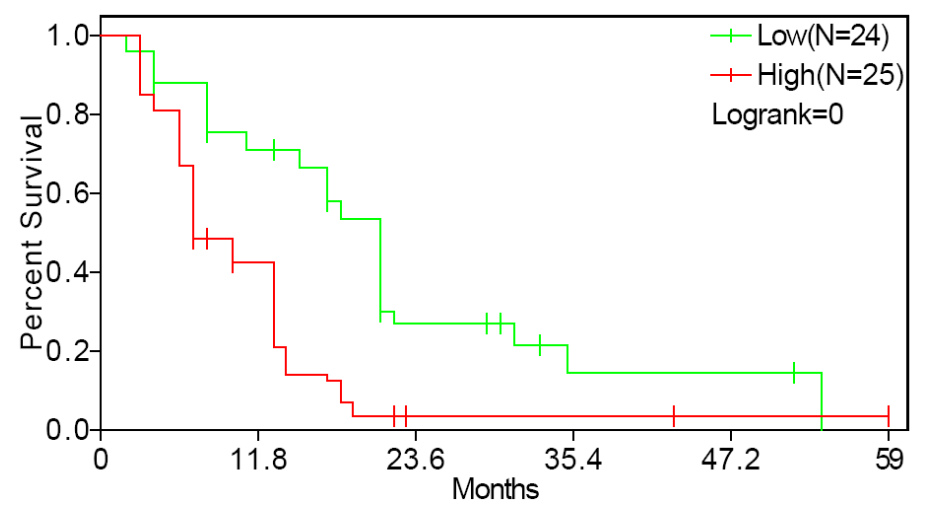


A

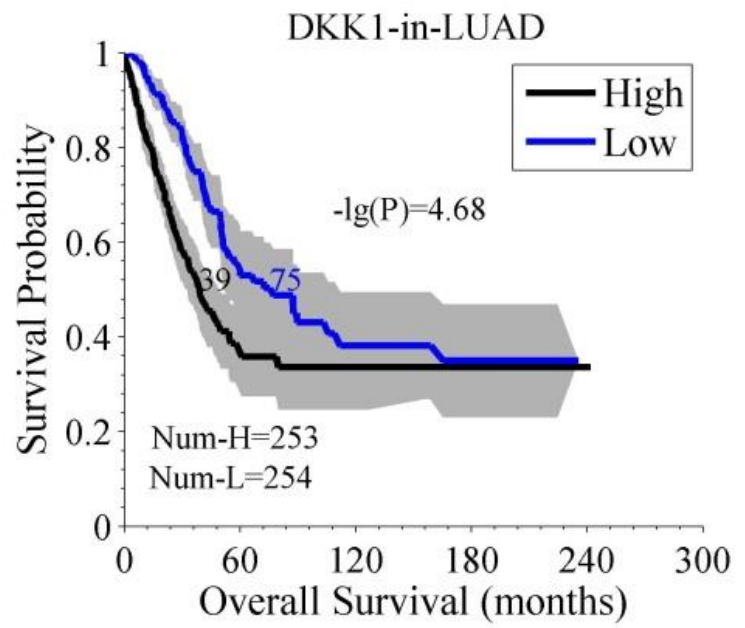

B

Figure S3

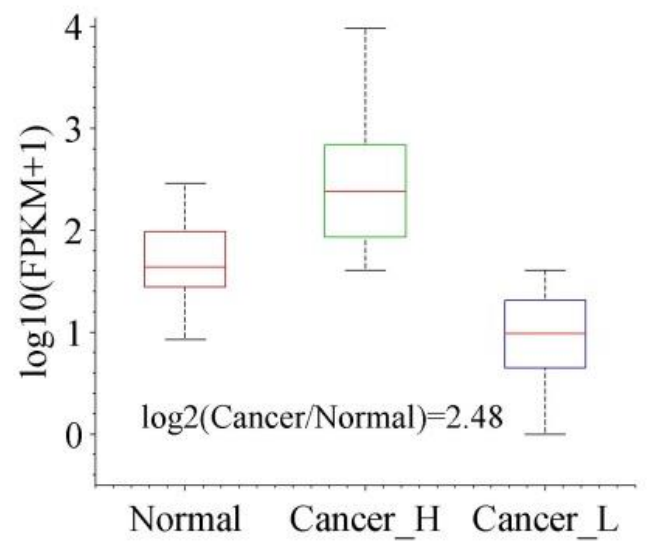

C

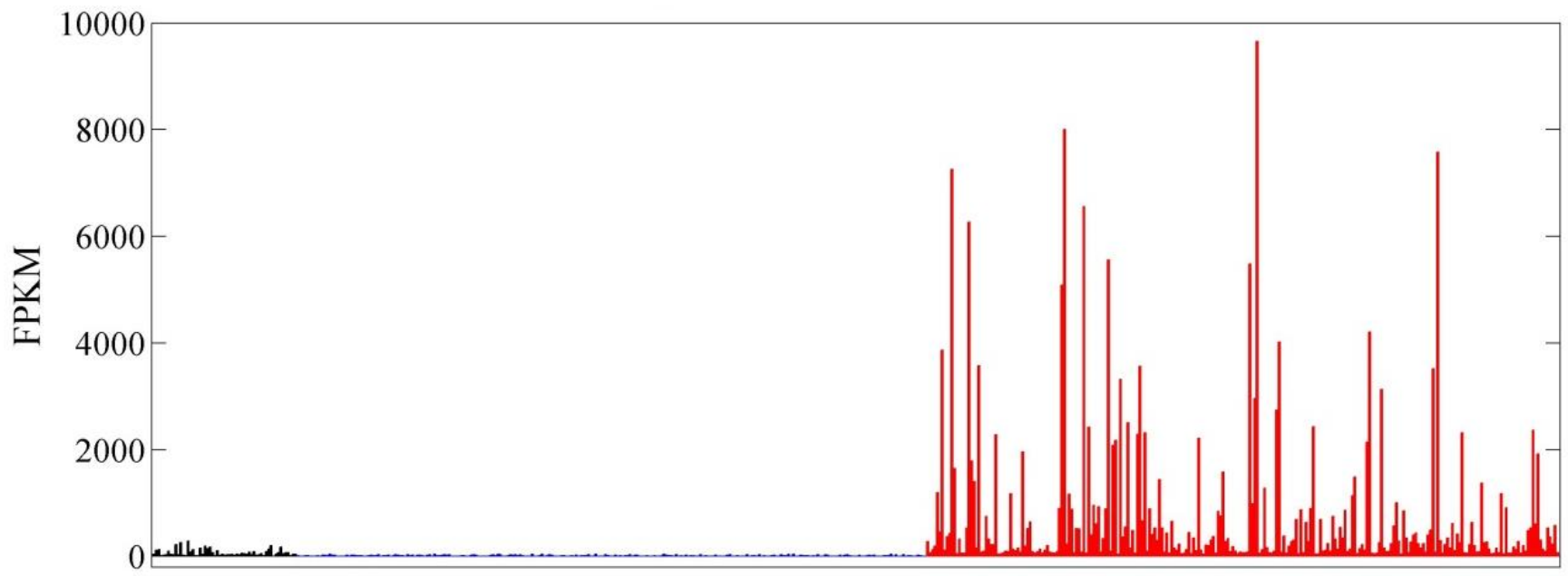

\begin{tabular}{|l|l||l}
\hline Normal & Low expression group & High expression group
\end{tabular}

D

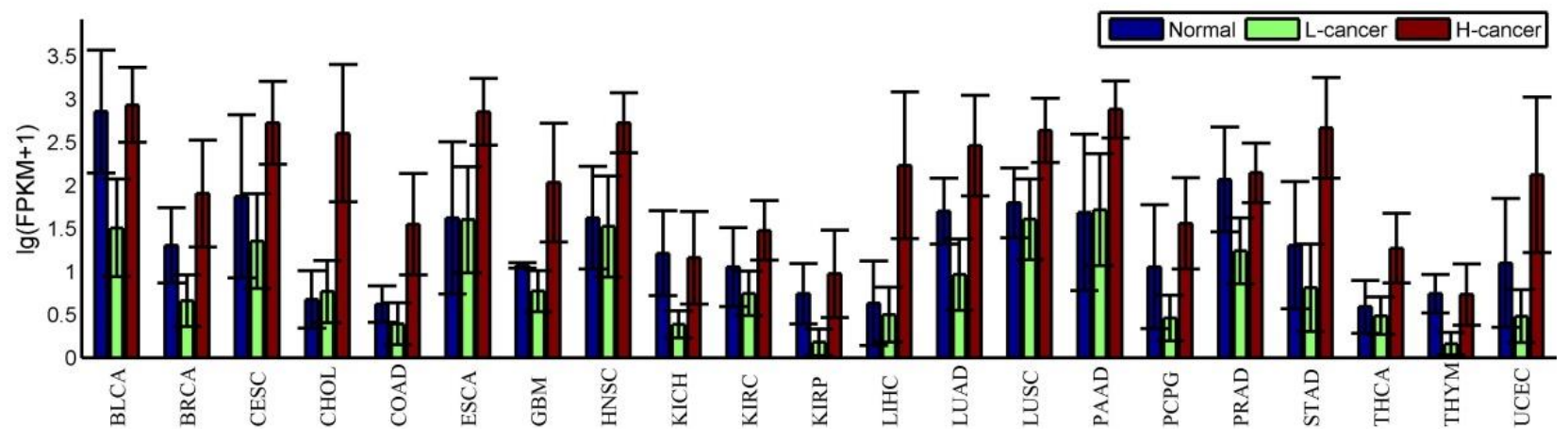




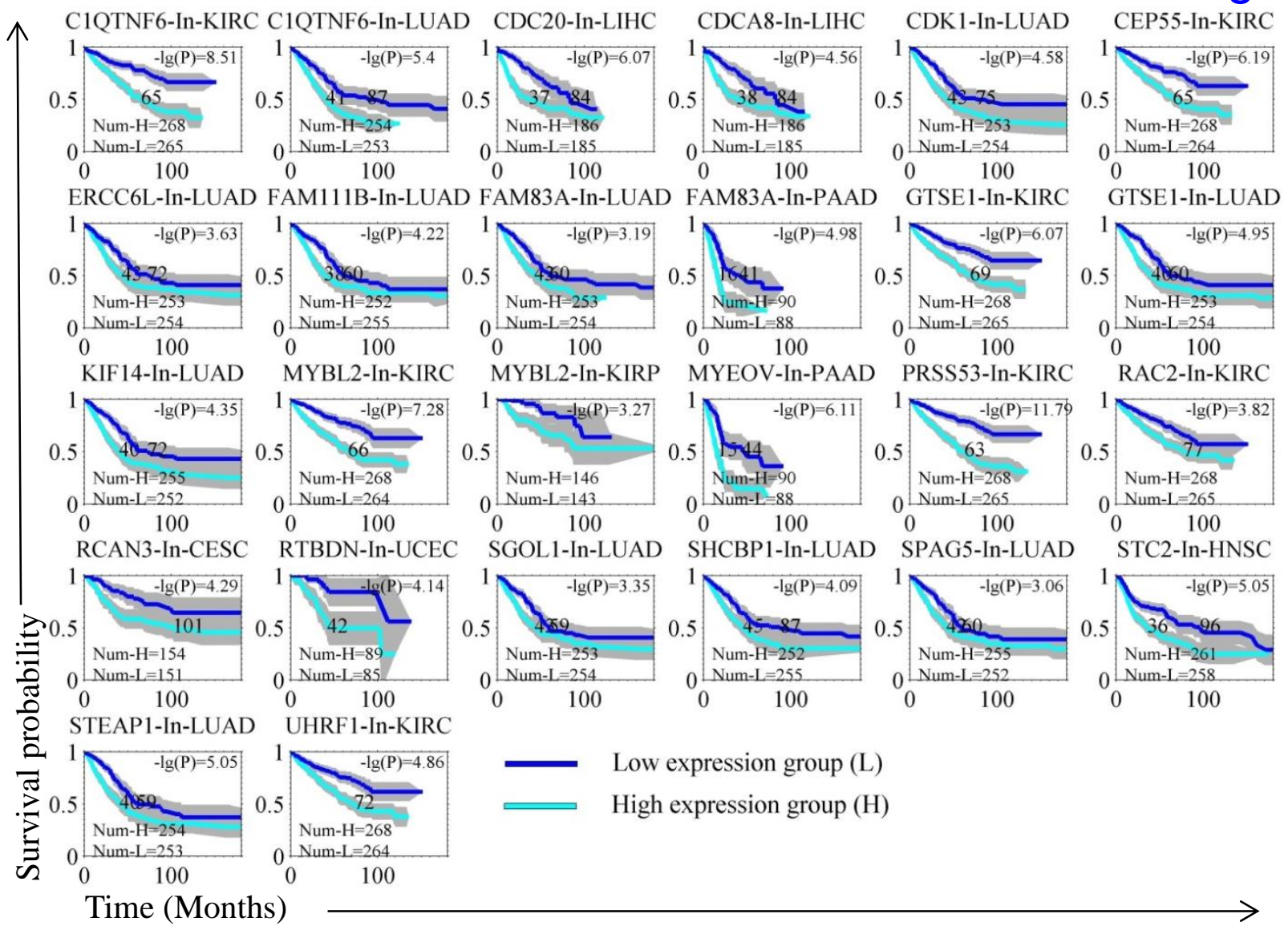

C1QTNF6-In-KIRC

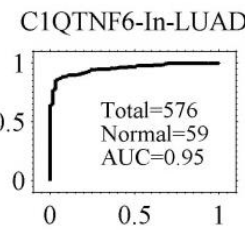

CDC20-In-LIHC

CDCA8-In-LIHC

CDK1-In-LUAD

CEP55-In-KIRC
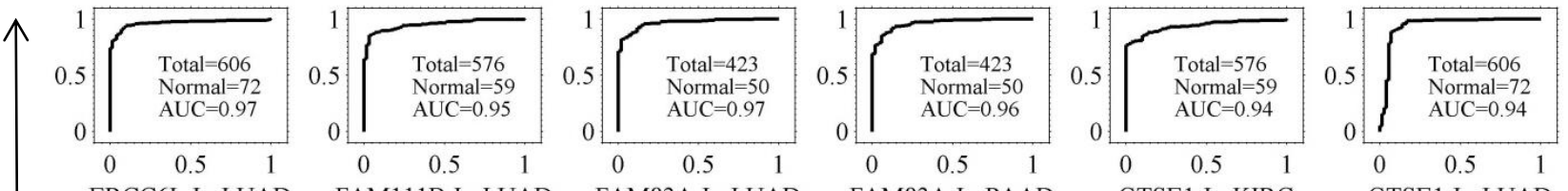

ERCC6L-In-LUAD

FAM111B-In-LUAD

FAM83A-In-LUAD

FAM83A-In-PAAD

GTSE1-In-KIRC
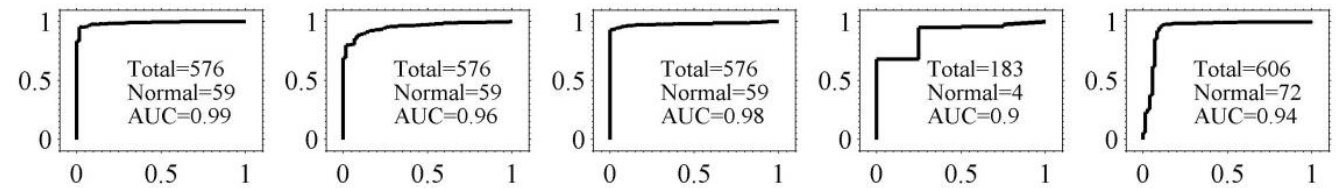

GTSE1-In-LUAD

KIF14-In-LUAD

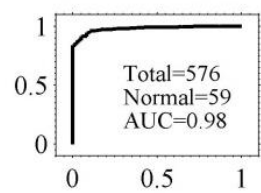

MYBL2-In-KIRC

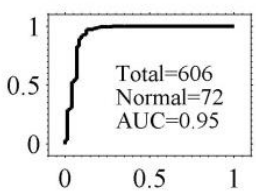

\section{MYBL2-In-KIRP}
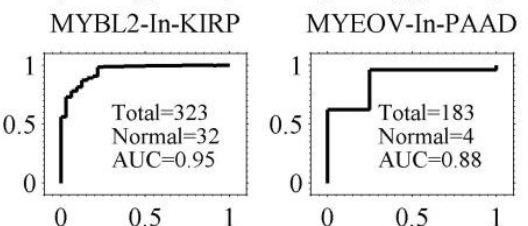

PRSS53-In-KIRC
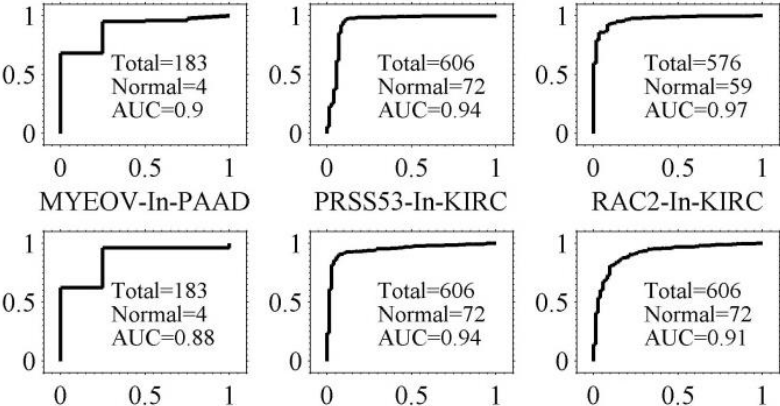

RCAN3-In-CESC

RTBDN-In-UCEC

SGOL1-In-LUAD

SHCBP1-In-LUAD

SPAG5-In-LUAD
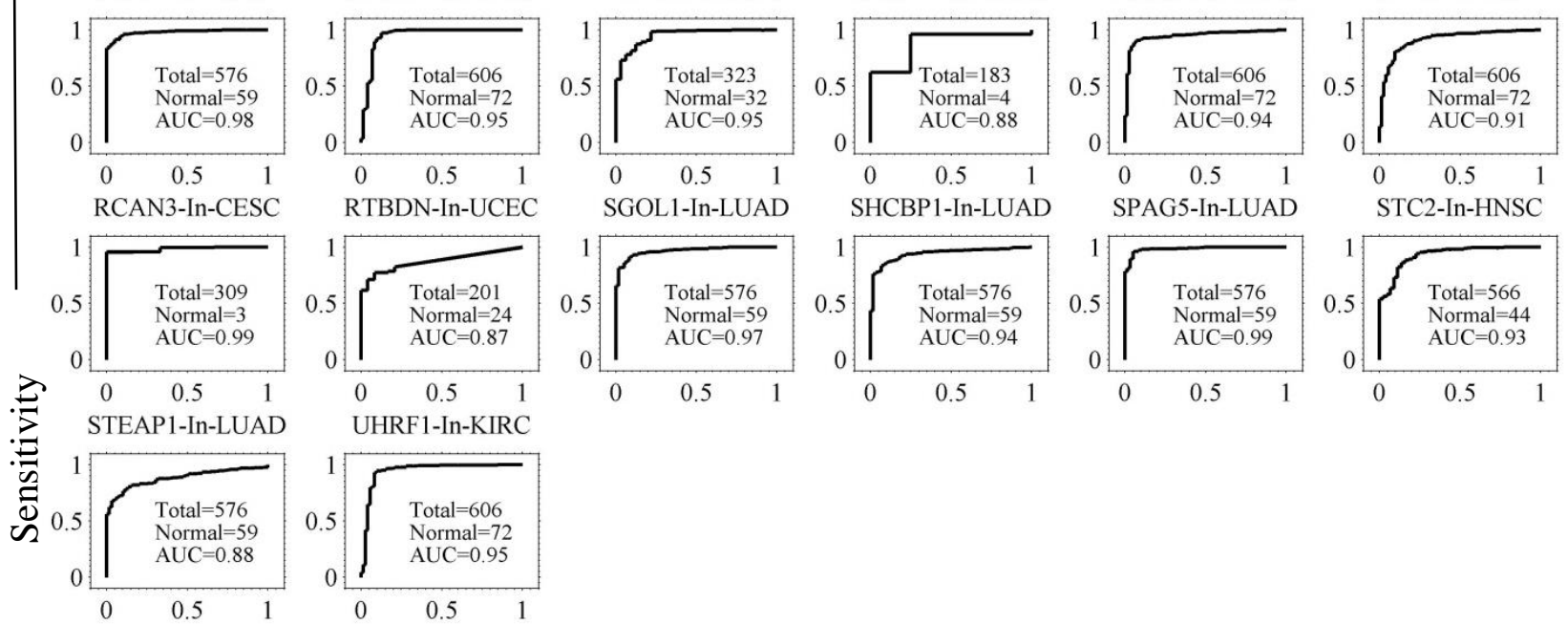

UHRF1-In-KIRC

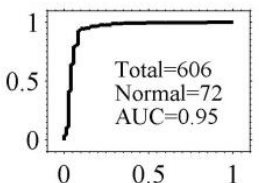




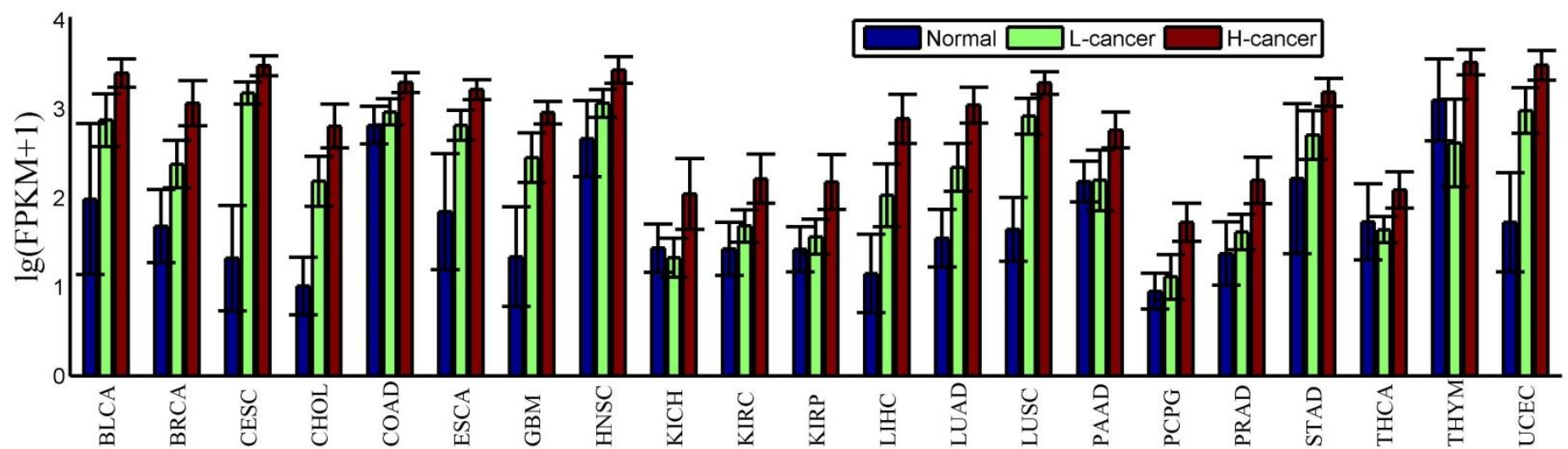

B

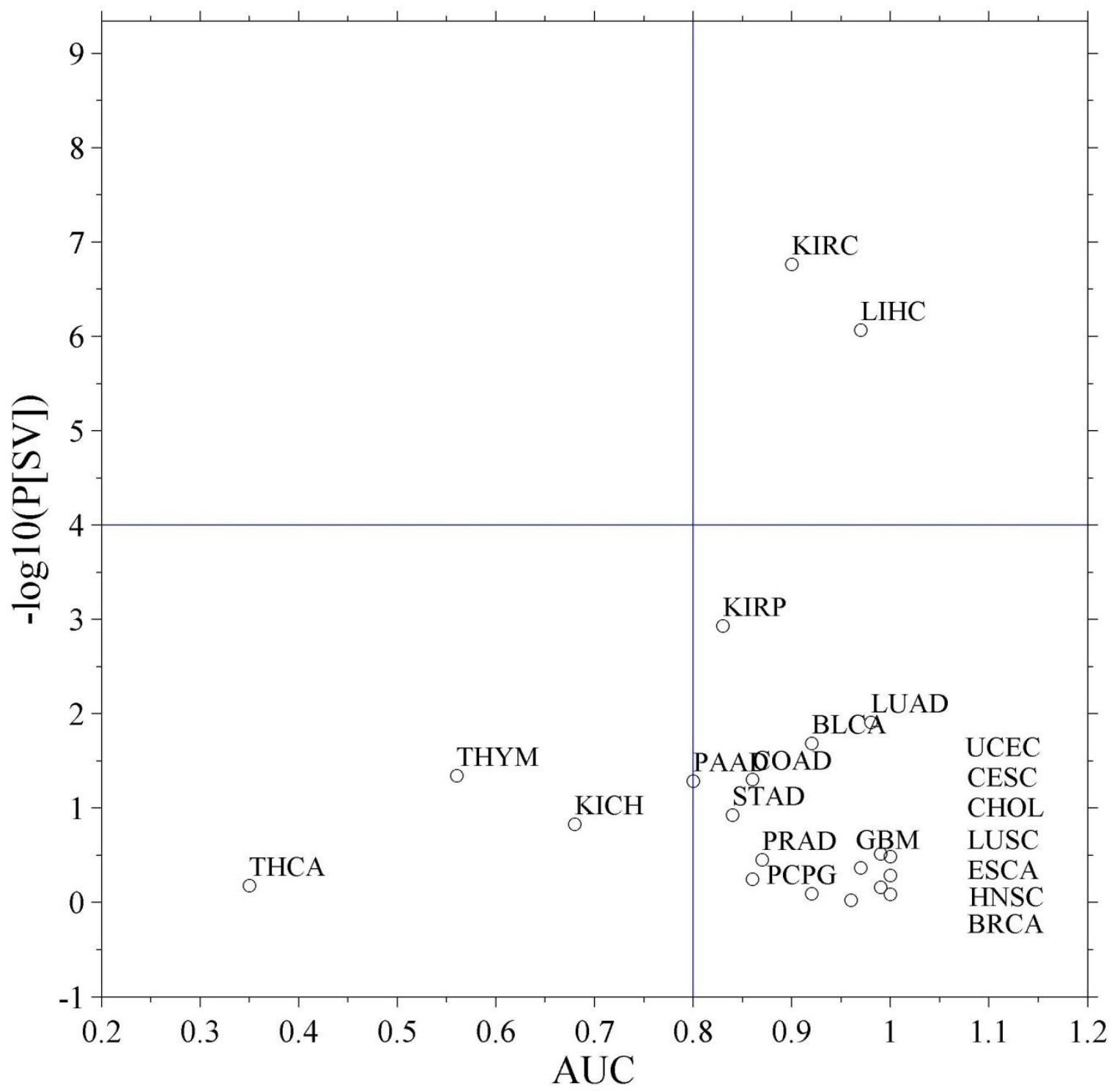




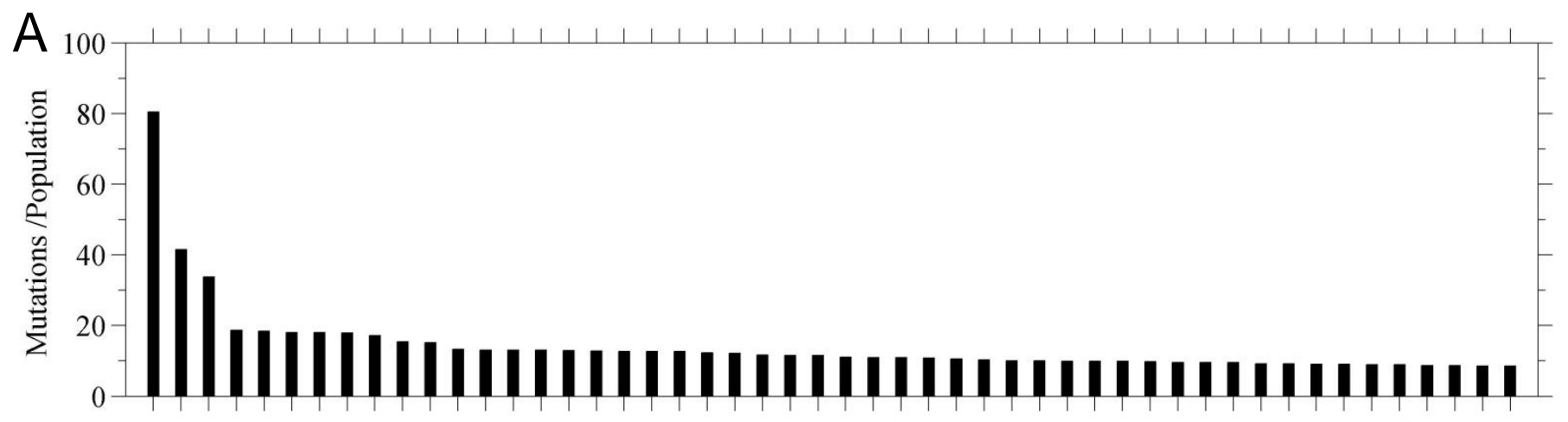

B

The top 200 genes ranked by the maximum mutation rate

\begin{tabular}{lrrrr}
\hline Term & Count & PValue & Benjamini & FDR \\
\hline IPR013783:Immunoglobulin-like fold & 24 & $1.1 \times 10^{-4}$ & 0.0016 & 0.16 \\
hsa04510:Focal adhesion & 12 & $2.1 \times 10^{-5}$ & 0.0034 & 0.026 \\
hsa05213:Endometrial cancer & 7 & $2.6 \times 10^{-5}$ & 0.0020 & 0.032 \\
hsa04512:ECM-receptor interaction & 8 & $5.9 \times 10^{-4}$ & 0.0030 & 0.071 \\
hsa04151:PI3K-Akt signaling pathway & 12 & $2.0 \times 10^{-3}$ & 0.033 & 2.30 \\
\hline
\end{tabular}

C

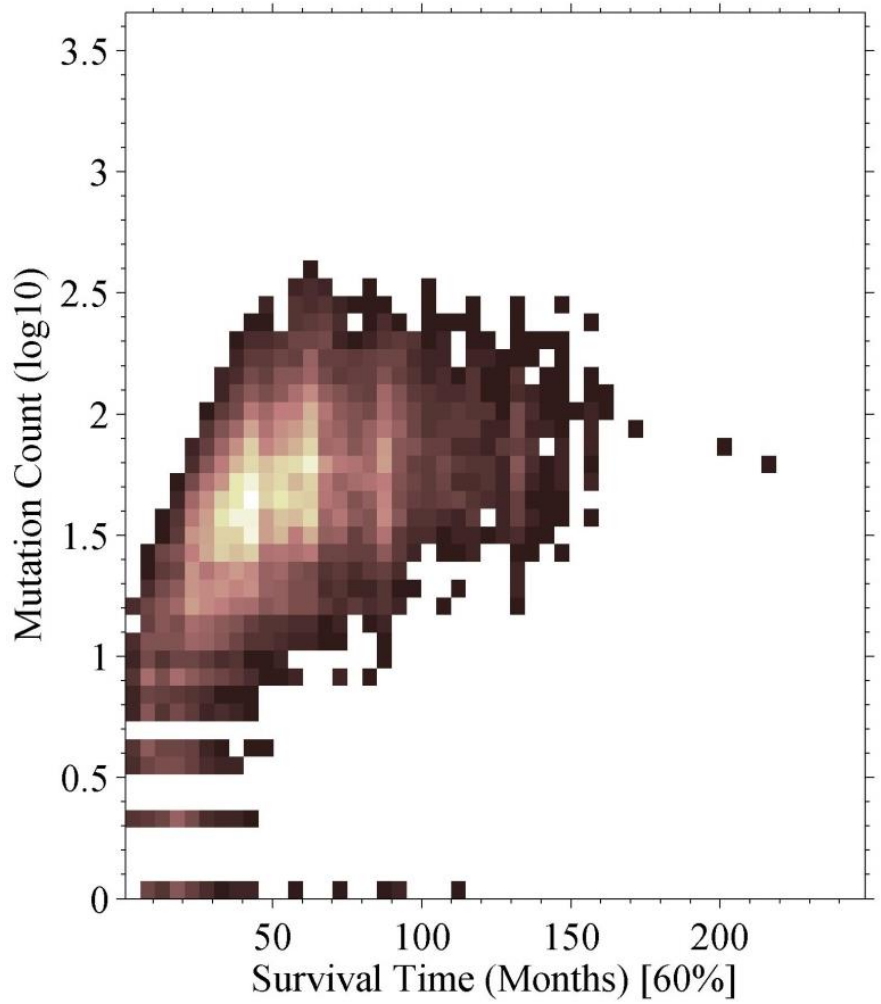

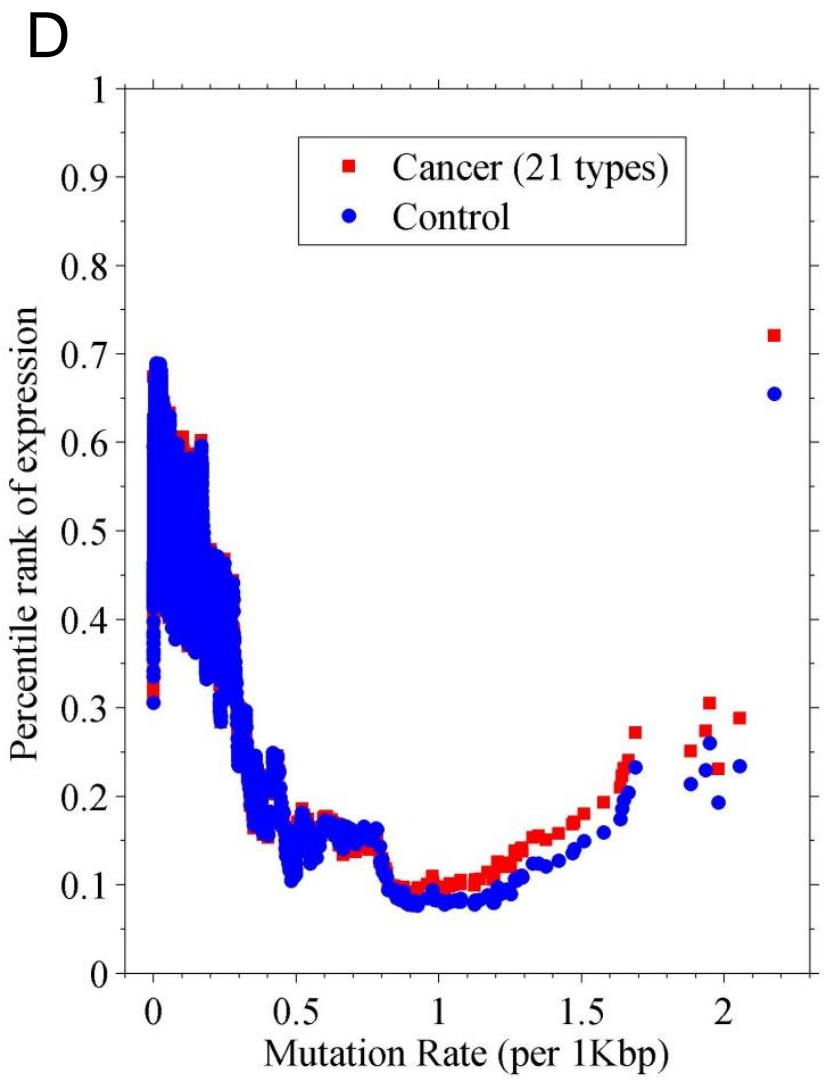




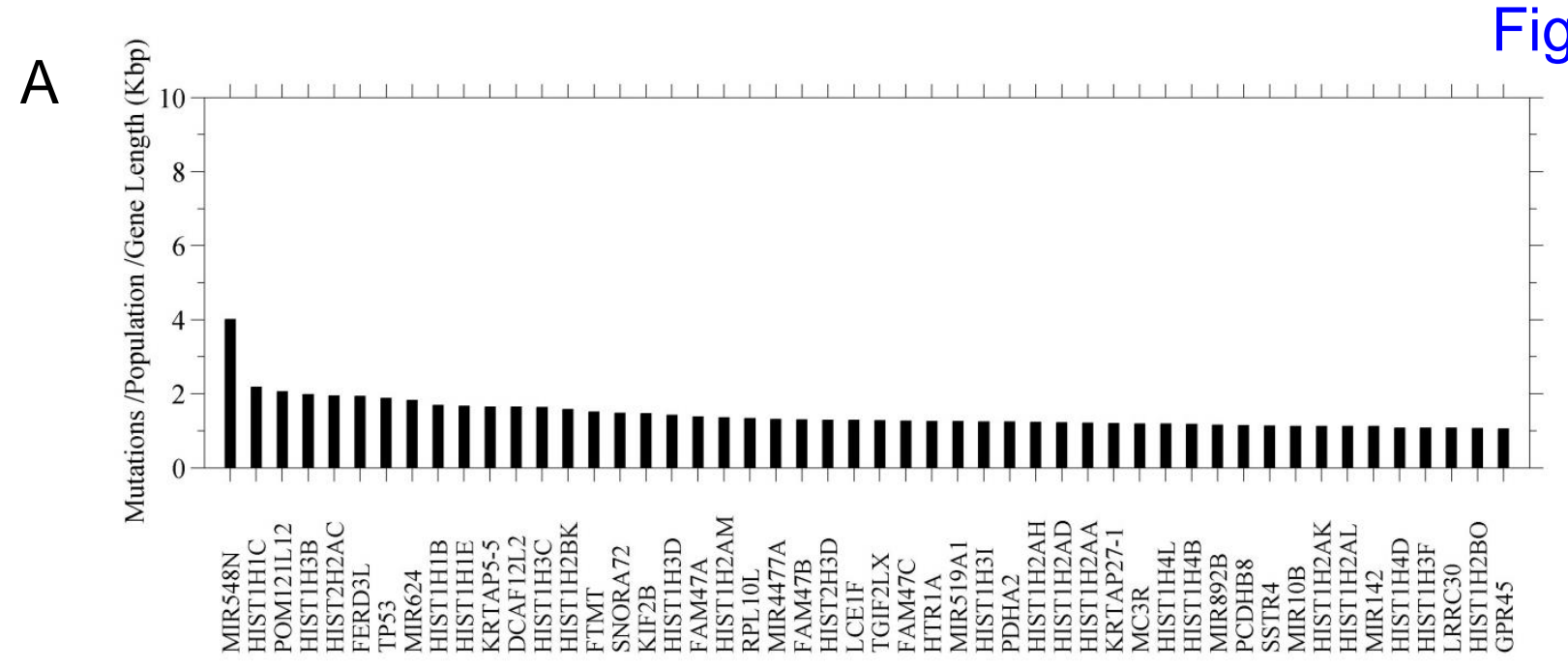

B
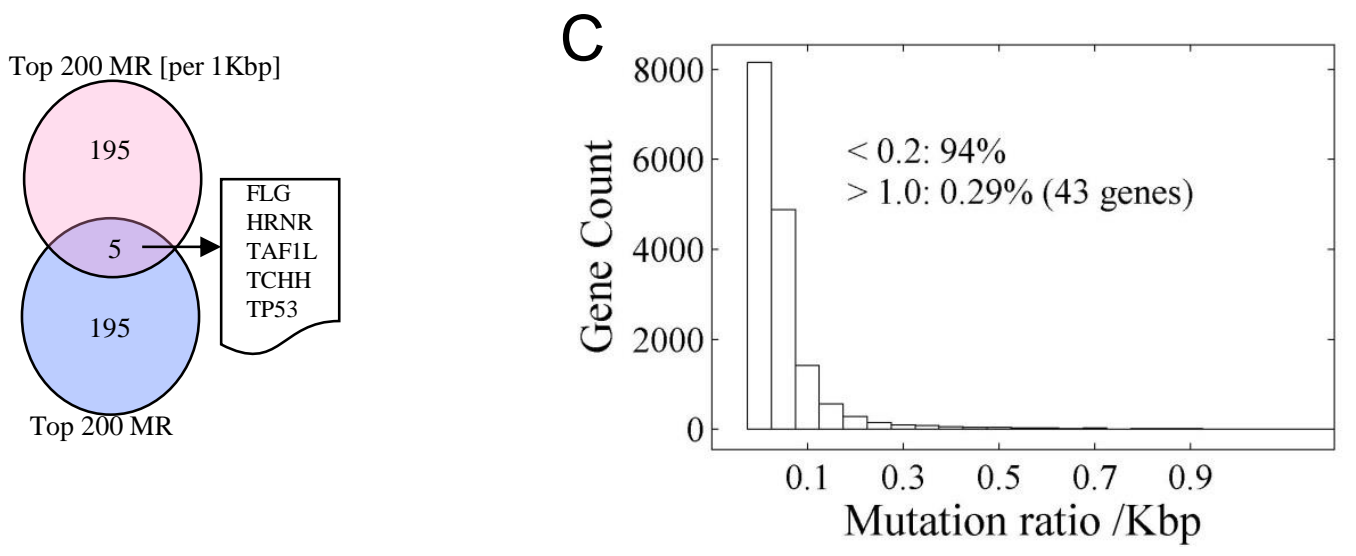

D The top 200 genes ranked by the normalized maximum mutation rate

GO:0000786 nucleosome $\left(\mathrm{fdr}=10^{-38.5}\right)$

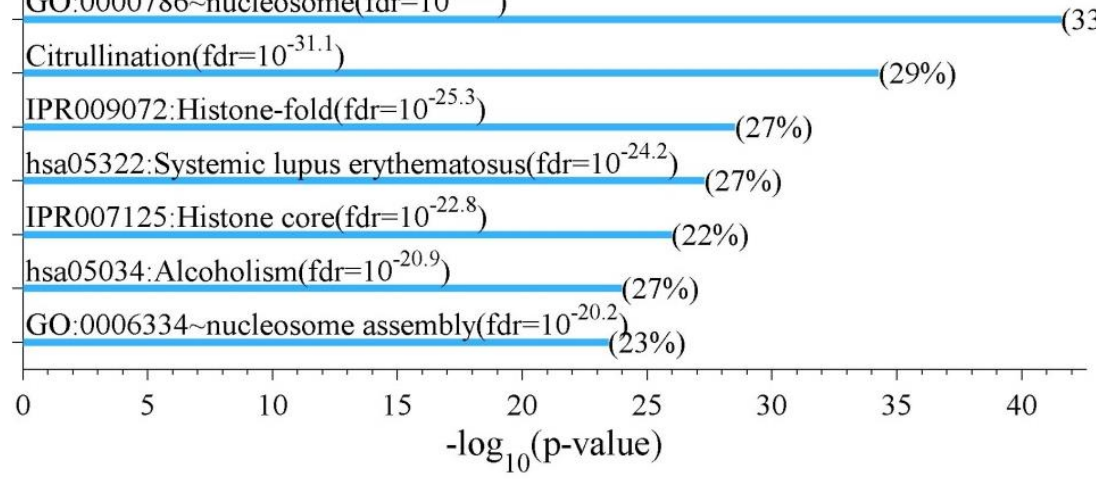

The top 200 genes whose mutations associate to the shortest survival time

E $\quad$ IPR013106:Immunoglobulin V-set $\left(\mathrm{fdr}=10^{-11.4}\right)$

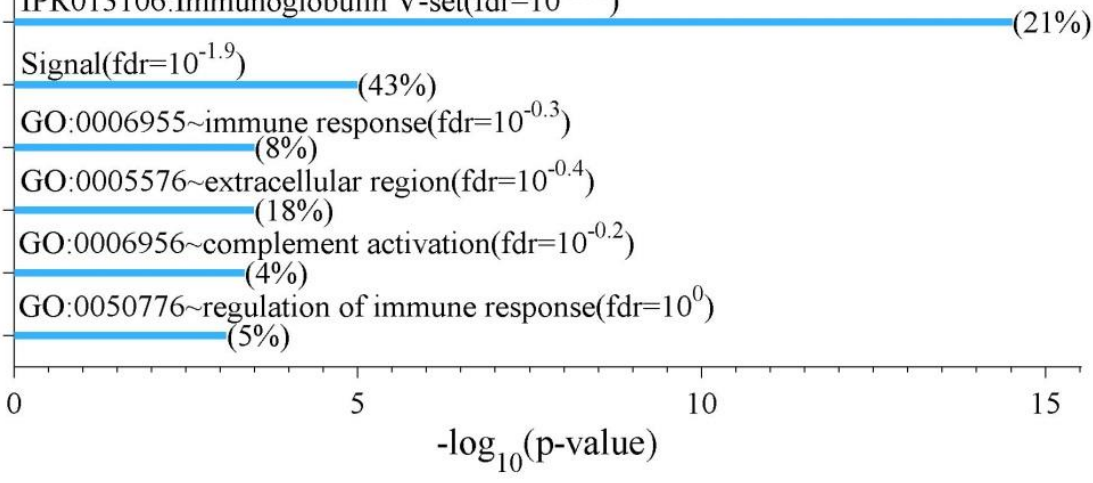

